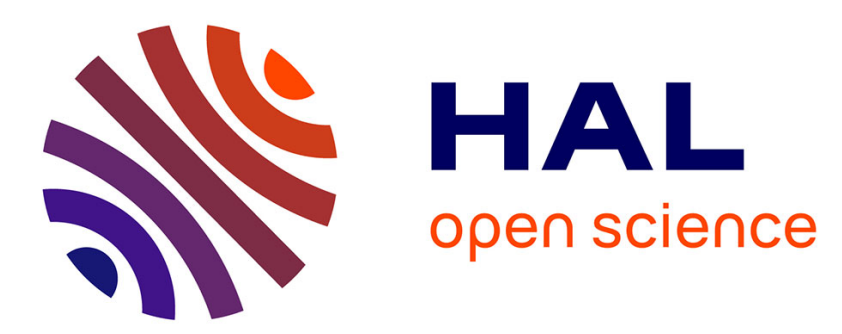

\title{
Pressure-velocity coupling allowing acoustic calculation in low Mach number flow
}

Yann Moguen, Tarik Kousksou, Pascal Bruel, Jan Vierendeels, Erik Dick

\section{To cite this version:}

Yann Moguen, Tarik Kousksou, Pascal Bruel, Jan Vierendeels, Erik Dick. Pressure-velocity coupling allowing acoustic calculation in low Mach number flow. Journal of Computational Physics, 2012, 231 (16), pp.5522-5541. 10.1016/j.jcp.2012.05.001 . hal-02358963

\section{HAL Id: hal-02358963 https://hal-univ-pau.archives-ouvertes.fr/hal-02358963}

Submitted on 13 Nov 2019

HAL is a multi-disciplinary open access archive for the deposit and dissemination of scientific research documents, whether they are published or not. The documents may come from teaching and research institutions in France or abroad, or from public or private research centers.
L'archive ouverte pluridisciplinaire HAL, est destinée au dépôt et à la diffusion de documents scientifiques de niveau recherche, publiés ou non, émanant des établissements d'enseignement et de recherche français ou étrangers, des laboratoires publics ou privés. 


\title{
Pressure-velocity coupling allowing acoustic calculation in low Mach number flow
}

\author{
Yann Moguen $^{\text {a, }}$, Tarik Kousksou ${ }^{\mathrm{b}}$, Pascal Bruel ${ }^{\mathrm{c}}$, Jan Vierendeels ${ }^{\text {a }}$ \\ and Erik Dick ${ }^{\text {a,* }}$

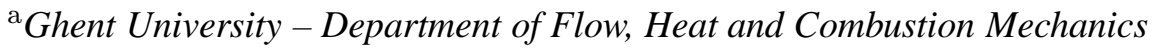 \\ Sint-Pietersnieuwstraat, 41 - 9000 Gent (Belgium) \\ ${ }^{\mathrm{b}}$ Université de Pau et des Pays de l'Adour Laboratoire SIAME and Inria CAGIRE Team \\ Bât. d'Alembert, rue Jules Ferry, BP 7511, 64075 Pau (France) \\ ${ }^{\mathrm{C}}$ CNRS, Université de Pau et des Pays de l'Adour LMAP UMR 5142 and Inria CAGIRE \\ Team \\ Avenue de l'Université, 64013 Pau (France)
}

\begin{abstract}
Low Mach number flow computation in co-located grid arrangement requires pressurevelocity coupling in order to prevent the checkerboard phenomenon. Two broad categories of pressure-velocity coupling methods for unsteady flows can be distinguished based on the time-step dependency of the coupling coefficient in the definition of the transporting velocity on a face of a control volume. As an example of the time-step independent category, the $\mathrm{AUSM}^{+}$-up scheme is studied. As an example of the second category, Rhie-Chow momentum interpolation methods are studied. Within the momentum interpolation techniques, again two broad categories can be distinguished based on the time-step dependency of the coupling coefficient used for unsteady flow computations, but when a steady state is reached. Variants of Rhie-Chow interpolation methods in each subcategory are studied on critical test cases. The result of the study is that for a good representation of unsteady flows containing acoustic information, the pressure-velocity coupling coefficient must explicitly depend on the time-step, but that the transporting velocity must become independent of the time-step when a steady state is reached.
\end{abstract}

Key words: Pressure-velocity coupling, Momentum Interpolation method, Low Mach number flow, Pressure correction, Acoustics

\footnotetext{
* Corresponding author. Tel.: +3292643301.

Email address: Erik. Dick@Ugent. be (Erik Dick).
} 


\section{Introduction}

Solving the compressible flow model instead of the incompressible - or else, the dilatable flow model - to simulate low Mach number flow, presents an important advantage: the acoustic field is resolved as well as the hydrodynamic one. For instance, this allows to get insight into the physical mechanisms of sound generation in the flow. However, a particular difficulty arises for low Mach number computations in finite volume formulation with co-located arrangement, since pressure-velocity coupling is then required to avoid the checkerboard decoupling phenomenon, which increases as the Mach number decreases. Albeit present in principle in the calculated field, the acoustic waves can then be damped so that no usable acoustic information is obtained. This holds even if small time-step and mesh spacing are used. Thus, when solving the compressible equations in low Mach number regime, special care has to be applied to introduce a pressure-velocity coupling that preserves meaningful acoustic information, and more generally, to calculate unsteady solutions satisfactorily.

Broadly, two large classes of pressure-velocity coupling can be distinguished in unsteady calculations. First, the pressure-velocity coupling can be introduced through a coefficient that does not depend explicitly on the time-step (see e.g. [4,8]). In the second class, the pressure-velocity coupling is explicitly time-step dependent in unsteady calculation (see e.g. $[2,5,6,11,15,20]$ ). Rhie-Chow interpolation, which consists in the construction of the transporting - or interface - velocity inspired by the method presented in Ref. [13] for steady calculations, belongs to this second class of methods. However, the interpretation of this interpolation method is not unique, as far as unsteady calculations are considered.

As pointed out by Pascau [11], an important property justifies the distinction between two groups of unsteady momentum interpolation methods. The distinctive property is whether or not the interpolation carried out for steady flows is recovered when a steady state is reached while the interpolation is carried out for un-

steady flows. Evidently, this property is strongly related to the time consistency of the scheme. If it is satisfied, the time-step independency of the steady state is guaranteed. For instance, this is the case for the interpolation procedures suggested in Refs. [6,11,20], but not for the ones suggested in Refs. [2,5,15]. Let us emphasize that, as clearly shown in Ref. [11], the time-step dependency of the steady state is not the consequence of the presence of the time-step as multiplying factor of the pressure gradient in the transporting velocity expression.

The present investigation focuses on the manner in which the pressure-velocity coupling should be carried out to allow high quality unsteady low Mach number flow calculations as well as steady ones. To compare the different possibilities of this coupling, belonging to the groups mentioned above, a common pressure correction algorithm is used. It is described in Sec. 2. The pressure-velocity coupling is intro- 
duced through the construction of the transporting velocity only. This velocity is unique in the flux terms when solving the basic conservation equations, for mass, momentum and energy. Various elements of its construction are detailed in Sec. 3. To probe the schemes, a series of one-dimensional problems and a two-dimensional problem, for which exact solutions exist or for which the physical interpretation is obvious, are chosen. The numerical experiments are presented in Sec. 4.

\section{Pressure correction algorithm}

In this section, the common features of the methods considered in the following are described. For simplicity, a one-dimensional flow of a perfect and ideal gas in a nozzle with a variable or constant section is considered. In the following, $x$ denotes the coordinate in the flow direction. The flow model is given by the Euler equations:

$$
\begin{array}{r}
\partial_{t}(\varrho S)+\partial_{x}(\varrho v S)=0 \\
\partial_{t}(\varrho v S)+\partial_{x}\left(\left(\varrho v^{2}+p\right) S\right)=p \mathrm{~d}_{x} S \\
\partial_{t}(\varrho E S)+\partial_{x}(\varrho v H S)=0 \\
E=e+\frac{1}{2} v^{2} \\
\varrho H=\varrho E+p \\
\varrho e=\frac{p}{\gamma-1}
\end{array}
$$

where $t, \varrho, p, v, e, E$ and $H$ represent time, density, pressure, velocity, internal energy, total energy and total enthalpy per unit mass, respectively. Furthermore, $\gamma$ denotes the ratio of the specific heats and $S$ the cross-section area of the nozzle. The $x$ axis along the nozzle is divided into $N$ cells of length $\Delta x$. A finite volume formulation in co-located arrangement is applied.

\subsection{Prediction-correction method}

In the solution procedure adopted, each time-step $n \rightarrow n+1$ is decomposed into iterations that take the following form, where the superscripts $\star$ and / denote predicted and correction quantities of each iteration $k$ (first iteration: $k=n$ ):

(1) Pre-estimation step: Generate a transporting velocity $v_{i+1 / 2}^{\mathrm{T}}$ on the cell face, that will be used in the following two steps. The transporting velocity is defined through an auxiliary density $\varrho^{\star \star}$ and velocity $v^{\star \star}$, that can be thought of as 'pre-predicted'. These are obtained from the continuity and momentum equations, where the cell face velocity $v_{i+1 / 2}^{k}$ and pressure $p_{i+1 / 2}^{k}$ are calcu- 
lated through a simple low Mach number adaptation of the $\mathrm{AUSM}^{+}$interpolation (see Sec. 2.2). For instance, the momentum equation is written as:

$$
\begin{aligned}
& \frac{1}{2}\left(3(\varrho v)_{i}^{\star \star}-4(\varrho v)_{i}^{n}+(\varrho v)_{i}^{n-1}\right) \\
& +\frac{\tau}{S_{i}}\left[\left\{(\varrho v)_{i}^{\star \star}+\frac{1}{2} \psi_{i}\left((\varrho v)^{k}\right)\left[(\varrho v)_{i}^{k}-(\varrho v)_{i-1}^{k}\right]\right\} v_{i+1 / 2}^{k} S_{i+1 / 2}\right. \\
& \left.\left.-\left\{(\varrho v)_{i-1}^{k}+\frac{1}{2} \psi_{i-1}\left((\varrho v)^{k}\right)\left[(\varrho v)_{i-1}^{k}-(\varrho v)_{i-2}^{k}\right)\right]\right\} v_{i-1 / 2}^{k} S_{i-1 / 2}\right] \\
& +\tau\left(p_{i+1 / 2}^{k}-p_{i-1 / 2}^{k}\right)=0
\end{aligned}
$$

where $v^{\mathrm{T}}$ is positive and $\tau$ is formally defined as $\Delta t / \Delta x$ and practically calculated as $\mathrm{CFL}_{v} / v_{\text {max }}$. Here, $\psi$ denotes a slope limiter, for instance Bounded Central (defined later), allowing to reach second-order accuracy in space, while the same order of accuracy in time is obtained by using the secondorder backward discretization. The construction of the transporting velocity will be detailed in Sec. 3. This transporting velocity is used in all equations in the prediction step. In the pre-estimation step, the boundary conditions are taken into account.

The pre-estimation step is introduced here in order to allow a unique definition of the transporting velocity in all equations in the prediction step. In most practical algorithms, this pre-estimation step is not used. This then has as consequence that the transporting velocity in the momentum equation differs from the transporting velocity in the equation used to derive the pressure field, which typically is the continuity equation. In the present study, we introduce the pre-estimation step in order to avoid phenomena related to differences in the transporting velocities in the different equations.

(2) Prediction step: With $p_{i}^{\star}=p_{i}^{k}$, calculate $\varrho_{i}^{\star}$ using

$$
\begin{aligned}
\frac{1}{2}\left(3 \varrho_{i}^{\star}-4 \varrho_{i}^{n}+\varrho_{i}^{n-1}\right)+ & \frac{\tau}{S_{i}}\left\{\left[\varrho_{i}^{\star}+\frac{1}{2} \psi_{i}\left(\varrho^{k}\right)\left(\varrho_{i}^{k}-\varrho_{i-1}^{k}\right)\right] v_{i+1 / 2}^{\mathrm{T}} S_{i+1 / 2}\right. \\
- & {\left.\left[\varrho_{i-1}^{\star}+\frac{1}{2} \psi_{i-1}\left(\varrho^{k}\right)\left(\varrho_{i-1}^{k}-\varrho_{i-2}^{k}\right)\right] v_{i-1 / 2}^{\mathrm{T}} S_{i-1 / 2}\right\}=0 }
\end{aligned}
$$

where the transporting velocity $v^{\mathrm{T}}$ is positive. A similar equation stands for momentum $(\varrho v)^{\star}$. From the predicted density, momentum and pressure, calculate the predicted values of total energy and total enthalpy.

(3) Correction step: Calculate the pressure correction $p^{\prime}$ by solving the energy equation in second-order accurate backward discretization form in time. Flux terms are expanded as

$$
(\varrho v H)_{i+1 / 2}^{k+1}=(\varrho H)_{i+1 / 2}^{\star} v_{i+1 / 2}^{\mathrm{T}}+H_{i+1 / 2}^{\star}(\varrho v)_{i+1 / 2}^{\prime}+(\varrho H)_{i+1 / 2}^{\prime} v_{i+1 / 2}^{\mathrm{T}}
$$

where $(\varrho H)_{i+1 / 2}^{\star}$ is upwinded in second-order accurate form as a convected quantity, as well as $H_{i+1 / 2}^{\star}$. Furthermore, neglecting the kinetic energy contribution, $(\varrho H)_{i+1 / 2}^{\prime}=\frac{\gamma}{\gamma-1} p_{i+1 / 2}^{\prime}$ and $(\varrho v)_{i+1 / 2}^{\prime}$ and $(\varrho v)_{i}^{\prime}$ are derived from the 
momentum equation according to the SIMPLE approximation method. This will be detailed in Sec. 2.3.

(4) Updates: $p_{i}^{k+1}=p_{i}^{\star}+p_{i}^{\prime}, \varrho_{i}^{k+1}=\varrho_{i}^{\star}+\left(\partial_{p} \varrho\right)_{i}^{\star} p_{i}^{\prime},(\varrho v)_{i}^{k+1}=(\varrho v)_{i}^{\star}+(\varrho v)_{i}^{\prime}$. The total energy, total enthalpy, the cell face pressure $p_{i+1 / 2}^{k+1}$ and velocity $v_{i+1 / 2}^{k+1}$ are finally updated.

\subsection{Cell face quantities in the pre-estimation stage}

For explanations on the $\mathrm{AUSM}^{+}$and $\mathrm{AUSM}^{+}$-up schemes, we refer to [7,8] and focus only on a low Mach number adaptation of $\mathrm{AUSM}^{+}$, by using the scaling function in the construction of the $\mathrm{AUSM}^{+}$-up scheme. The notation $L$ or $R$, which refers to the left or right side of the face $i+1 / 2$, is adopted since an extrapolation technique will be used in the following. Thus, a Mach number on the side $S$ is defined as

$$
M_{S}=\frac{v_{S}}{c_{i+1 / 2}} \quad, \quad S=L, R
$$

where $c_{i+1 / 2}$ is the interface speed of sound defined in Ref. [7]. A mean Mach number at the face $i+1 / 2$ is also defined,

$$
\bar{M}_{i+1 / 2}=\sqrt{\frac{\left(v_{L}\right)^{2}+\left(v_{R}\right)^{2}}{2 c_{i+1 / 2}^{2}}}
$$

and a reference Mach number $M_{0, i+1 / 2}$ by

$$
M_{0, i+1 / 2}^{2}=\min \left\{1, \max \left\{\bar{M}_{i+1 / 2}^{2}, \mathrm{M}_{\text {co }}^{2}\right\}\right\}
$$

where $M_{c o}$ is a cut-off Mach number that belongs to $\mathcal{O}\left(\mathrm{M}_{\infty}\right)$. The scaling function suggested in Ref. [8] is

$$
f(M)=M(2-M) .
$$

The use of this function permits the proper asymptotic behaviour of the pressure dissipation term for $\mathrm{M} \searrow 0$ in the face velocity (see Ref. [8]), defined by the following expressions:

$$
\begin{array}{r}
M_{(1)}^{ \pm}(M)=\frac{1}{2}(M \pm|M|) \\
M_{(4)}^{ \pm}(M)= \pm \frac{1}{4}(M \pm 1)^{2} \pm \frac{1}{8}\left(M^{2}-1\right)^{2} \\
P_{(0)}^{ \pm}(M)=M_{(1)}^{ \pm}(M) / M \\
P_{(5)}^{ \pm}(M)=\frac{1}{4}(M \pm 1)^{2}(2 \mp M) \pm \frac{3}{16}\left(5\left(f\left(M_{0}\right)\right)^{2}-4\right) M\left(M^{2}-1\right)^{2}
\end{array}
$$




$$
\begin{aligned}
& \mathcal{M}^{ \pm}(M)= \begin{cases}M_{(1)}^{ \pm}(M), & |M| \geq 1 \\
M_{(4)}^{ \pm}(M), & |M|<1\end{cases} \\
& \mathcal{P}^{ \pm}(M)= \begin{cases}P_{(0)}^{ \pm}(M), & |M| \geq 1 \\
P_{(5)}^{ \pm}(M), & |M|<1\end{cases} \\
& p_{i+1 / 2}=\mathcal{P}^{+}\left(M_{L}\right) p_{L}+\mathcal{P}^{-}\left(M_{R}\right) p_{R} \\
& M_{i+1 / 2}=\mathcal{M}^{+}\left(M_{L}\right)+\mathcal{M}^{-}\left(M_{R}\right) \\
& v_{i+1 / 2}=c_{i+1 / 2} M_{i+1 / 2} .
\end{aligned}
$$

To reach second-order accuracy in space, the primitive variables $p, \varrho$ and $v$ used in the $\mathrm{AUSM}^{+}$scheme are extrapolated at the face $i+1 / 2$ according to

$$
\phi_{L}=\phi_{i}+\frac{1}{2} \psi_{i}(\phi)\left(\phi_{i}-\phi_{i-1}\right) \quad, \quad \phi_{R}=\phi_{i+1}-\frac{1}{2} \psi_{i+1}(\phi)\left(\phi_{i+1}-\phi_{i}\right)
$$

where $\psi$ denotes a slope limiter. Practically, MinMod or Bounded Central were chosen. For instance, for Bounded Central:

$$
\psi_{i}(\theta)\left(\theta_{i}-\theta_{i-1}\right)=\operatorname{BC}\left(\theta_{i}-\theta_{i-1}, \theta_{i+1}-\theta_{i}\right)
$$

where

$$
\mathrm{BC}(a, b)=\operatorname{MinMod}(b, 2 a)
$$

and

$$
\operatorname{MinMod}(a, b)=\frac{\operatorname{sign}(a)+\operatorname{sign}(b)}{2} \min \{|a|,|b|\}
$$

\subsection{Energy-based pressure correction equation}

As in every algorithm that belongs to the SIMPLE family, a relationship between momentum correction and pressure correction has to be constructed. First, the momentum is predicted implicitly with the second-order upwind discretization for the convected quantity $\varrho v$ and the second-order backward discretization in time,

$$
\begin{aligned}
& \frac{1}{2}\left\{3(\varrho v)_{i}^{\star}-4(\varrho v)_{i}^{n}+(\varrho v)_{i}^{n-1}\right\} \\
& +\frac{\tau}{S_{i}}\left[\left\{(\varrho v)_{i}^{\star}+\frac{1}{2} \psi_{i}\left((\varrho v)^{k}\right)\left[(\varrho v)_{i}^{k}-(\varrho v)_{i-1}^{k}\right]\right\} v_{i+1 / 2}^{\mathrm{T}} S_{i+1 / 2}\right. \\
& \left.-\left\{(\varrho v)_{i-1}^{\star}+\frac{1}{2} \psi_{i-1}\left((\varrho v)^{k}\right)\left[(\varrho v)_{i-1}^{k}-(\varrho v)_{i-2}^{k}\right]\right\} v_{i-1 / 2}^{\mathrm{T}} S_{i-1 / 2}\right] \\
& +\tau\left(p_{i+1 / 2}^{k}-p_{i-1 / 2}^{k}\right)=0 .
\end{aligned}
$$


Substracting Eq. (8) from the one satisfied by the momentum at the iteration $k+1$ when the same discretization procedure has been applied,

$$
\begin{aligned}
& \frac{1}{2}\left\{3(\varrho v)_{i}^{k+1}-4(\varrho v)_{i}^{n}+(\varrho v)_{i}^{n-1}\right\} \\
& +\frac{\tau}{S_{i}}\left[\left\{(\varrho v)_{i}^{k+1}+\frac{1}{2} \psi_{i}\left((\varrho v)^{k+1}\right)\left[(\varrho v)_{i}^{k+1}-(\varrho v)_{i-1}^{k+1}\right]\right\} v_{i+1 / 2}^{k+1} S_{i+1 / 2}\right. \\
& \left.-\left\{(\varrho v)_{i-1}^{k+1}+\frac{1}{2} \psi_{i-1}\left((\varrho v)^{k+1}\right)\left[(\varrho v)_{i-1}^{k+1}-(\varrho v)_{i-2}^{k+1}\right]\right\} v_{i-1 / 2}^{k+1} S_{i-1 / 2}\right] \\
& +\tau\left(p_{i+1 / 2}^{k+1}-p_{i-1 / 2}^{k+1}\right)=0
\end{aligned}
$$

the following relation is obtained,

$$
\begin{aligned}
& \frac{3}{2}(\varrho v)_{i}^{\prime}+\frac{\tau}{S_{i}}\left\{\left[\left\{(\varrho v)_{i}^{k+1}+\frac{1}{2} \psi_{i}\left((\varrho v)^{k+1}\right)\left[(\varrho v)_{i}^{k+1}-(\varrho v)_{i-1}^{k+1}\right]\right\} v_{i+1 / 2}^{k+1}\right.\right. \\
& \left.-\left\{(\varrho v)_{i}^{\star}+\frac{1}{2} \psi_{i}\left((\varrho v)^{k}\right)\left[(\varrho v)_{i}^{k}-(\varrho v)_{i-1}^{k}\right]\right\} v_{i+1 / 2}^{\mathrm{T}}\right] S_{i+1 / 2} \\
& -\left[\left\{(\varrho v)_{i-1}^{k+1}+\frac{1}{2} \psi_{i-1}\left((\varrho v)^{k+1}\right)\left[(\varrho v)_{i-1}^{k+1}-(\varrho v)_{i-2}^{k+1}\right]\right\} v_{i-1 / 2}^{k+1}\right. \\
& \left.\left.-\left\{(\varrho v)_{i-1}^{\star}+\frac{1}{2} \psi_{i-1}\left((\varrho v)^{k}\right)\left[(\varrho v)_{i-1}^{k}-(\varrho v)_{i-2}^{k}\right]\right\} v_{i-1 / 2}^{\mathrm{T}}\right] S_{i-1 / 2}\right\} \\
& +\tau\left(p_{i+1 / 2}^{\prime}-p_{i-1 / 2}^{\prime}\right)=0
\end{aligned}
$$

which is approximated by:

$$
\begin{gathered}
\frac{3}{2}(\varrho v)_{i}^{\prime}=-\frac{\tau}{S_{i}}\left[\left\{(\varrho v)_{i}^{\prime}+\frac{1}{2} \psi_{i}\left((\varrho v)^{k}\right)\left[(\varrho v)_{i}^{\prime}-(\varrho v)_{i-1}^{\prime}\right]\right\} v_{i+1 / 2}^{\mathrm{T}} S_{i+1 / 2}\right. \\
\left.-\left\{(\varrho v)_{i-1}^{\prime}+\frac{1}{2} \psi_{i-1}\left((\varrho v)^{k}\right)\left[(\varrho v)_{i-1}^{\prime}-(\varrho v)_{i-2}^{\prime}\right]\right\} v_{i-1 / 2}^{\mathrm{T}} S_{i-1 / 2}\right] \\
-\tau\left(p_{i+1 / 2}^{\prime}-p_{i-1 / 2}^{\prime}\right) .
\end{gathered}
$$

For effective computation, the slope limiter term in Eq. (9) is replaced by the second-order upwind form. According to the SIMPLE approximation principle, a mass flux correction on the face is deduced from Eq. (9), as

$$
(\varrho v)_{i+1 / 2, M}^{\prime}=-\frac{2}{3} \tau\left(p_{i+1}^{\prime}-p_{i}^{\prime}\right)
$$

where the index $M$ denotes the momentum equation origin of this expression. However, let us leave the possibility open for a more general mass flux correction expression, which may take into account a contribution of the numerical flux function used, so that:

$$
(\varrho v)_{i+1 / 2}^{\prime}=(\varrho v)_{i+1 / 2, M}^{\prime}+(\varrho v)_{i+1 / 2, F}^{\prime}
$$

where

$$
(\varrho v)_{i+1 / 2, F}^{\prime}=-\kappa_{i+1 / 2}\left(p_{i+1}^{\prime}-p_{i}^{\prime}\right) .
$$


The value of the non negative coefficient $\kappa$ will be detailed in Sec. 3, according to the pressure-velocity coupling to be chosen.

To compute the pressure correction $p^{\prime}$, a key role is assigned to the energy equation. Again, in order to achieve second-order accuracy in time, the second-order backward scheme is used:

$$
\begin{aligned}
& \frac{1}{2}\left\{3(\varrho E)_{i}^{k+1}-4(\varrho E)_{i}^{n}+(\varrho E)_{i}^{n-1}\right\} \\
& +\frac{\tau}{S_{i}}\left\{(\varrho v H)_{i+1 / 2}^{k+1} S_{i+1 / 2}-(\varrho v H)_{i-1 / 2}^{k+1} S_{i-1 / 2}\right\}=0
\end{aligned}
$$

The total energy is expanded as

$$
(\varrho E)_{i}^{k+1}=(\varrho E)_{i}^{\star}+\left(\partial_{p}(\varrho e)\right)_{i}^{\star} p_{i}^{\prime} .
$$

In the case of a perfect gas,

$$
\left(\partial_{p}(\varrho e)\right)_{i}^{\star}=\frac{1}{\gamma-1}
$$

Neglecting the kinetic energy contribution, the total enthalpy correction at the face is written as:

$$
(\varrho H)_{i+1 / 2}^{\prime}=\frac{\gamma}{\gamma-1} p_{i+1 / 2}^{\prime}
$$

In the flux terms, the pressure correction is interpolated using the $\mathrm{AUSM}^{+}$adaptation described in Sec. 2.2:

$$
p_{i+1 / 2}^{\prime}=\mathcal{P}^{+}\left(M_{i}^{\star}\right) p_{i}^{\prime}+\mathcal{P}^{-}\left(M_{i+1}^{\star}\right) p_{i+1}^{\prime} .
$$

Thus, the energy equation (12) becomes

$$
C_{i-1} p_{i-1}^{\prime}+C_{i} p_{i}^{\prime}+C_{i+1} p_{i+1}^{\prime}=\Sigma_{i}
$$

where

$$
\begin{aligned}
& C_{i-1}=-\frac{\tau}{S_{i}}\left[\frac{\gamma}{\gamma-1} \mathcal{P}^{+}\left(M_{i-1}^{\star}\right) v_{i-1 / 2}^{\mathrm{T}}+\left(\kappa_{i-1 / 2}+\frac{2}{3} \tau\right) H_{i-1 / 2}^{\star}\right] S_{i-1 / 2} \\
& C_{i}=\frac{3}{2(\gamma-1)}+\frac{\tau}{S_{i}}\left\{\left[\frac{\gamma}{\gamma-1} \mathcal{P}^{+}\left(M_{i}^{\star}\right) v_{i+1 / 2}^{\mathrm{T}}+\left(\kappa_{i+1 / 2}+\frac{2}{3} \tau\right) H_{i+1 / 2}^{\star}\right] S_{i+1 / 2}\right. \\
& \left.-\left[\frac{\gamma}{\gamma-1} \mathcal{P}^{-}\left(M_{i}^{\star}\right) v_{i-1 / 2}^{\mathrm{T}}-\left(\kappa_{i-1 / 2}+\frac{2}{3} \tau\right) H_{i-1 / 2}^{\star}\right] S_{i-1 / 2}\right\} \\
& C_{i+1}=\frac{\tau}{S_{i}}\left[\frac{\gamma}{\gamma-1} \mathcal{P}^{-}\left(M_{i+1}^{\star}\right) v_{i+1 / 2}^{\mathrm{T}}-\left(\kappa_{i+1 / 2}+\frac{2}{3} \tau\right) H_{i+1 / 2}^{\star}\right] S_{i+1 / 2}
\end{aligned}
$$


and

$$
\begin{aligned}
\Sigma_{i}=-\frac{1}{2}\left\{3(\varrho E)_{i}^{\star}\right. & \left.-4(\varrho E)_{i}^{n}+(\varrho E)_{i}^{n-1}\right\} \\
& -\frac{\tau}{S_{i}}\left\{(\varrho H)_{i+1 / 2}^{\star} v_{i+1 / 2}^{\mathrm{T}} S_{i+1 / 2}-(\varrho H)_{i-1 / 2}^{\star} v_{i-1 / 2}^{\mathrm{T}} S_{i-1 / 2}\right\} .
\end{aligned}
$$

\section{Transporting velocity construction}

To avoid the checkerboard decoupling phenomenon that may arise at low Mach number when a co-located arrangement is used, the transporting velocity should be constructed such as to couple the pressure and the velocity. Examples of such couplings are considered in this section: a transporting velocity obtained by the $\mathrm{AUSM}^{+}$-up interpolation and several alternatives of Rhie-Chow interpolation.

\subsection{AUSM $^{+}$-up cell-face velocity}

$\mathrm{AUSM}^{+}$-up is an extension of $\mathrm{AUSM}^{+}$, that consists in the addition of pressurevelocity coupling terms in the expressions of the pressure and the Mach number at the interface (see Eqs. (5)-(6)). Eq. (5) is replaced by:

$$
\begin{aligned}
p_{i+1 / 2}=\mathcal{P}^{+} & \left(M_{L}\right) p_{L}+\mathcal{P}^{-}\left(M_{R}\right) p_{R} \\
& \quad-K_{v} \mathcal{P}^{+}\left(M_{L}\right) \mathcal{P}^{-}\left(M_{R}\right)\left(\varrho_{L}+\varrho_{R}\right) f\left(M_{i+1 / 2}\right) c_{i+1 / 2}\left(v_{R}-v_{L}\right)
\end{aligned}
$$

where $K_{v}$ is a positive constant. Moreover, instead of Eq. (6), the Mach number at the interface is expressed as

$$
\begin{aligned}
M_{i+1 / 2}=\mathcal{M}^{+}\left(M_{L}\right) & +\mathcal{M}^{-}\left(M_{R}\right) \\
& \quad-\frac{K_{p}}{f\left(M_{0, i+1 / 2}\right)} \max \left\{1-\left(\bar{M}_{i+1 / 2}\right)^{2} \sigma, 0\right\} \frac{p_{R}-p_{L}}{\varrho_{i+1 / 2} c_{i+1 / 2}^{2}}
\end{aligned}
$$

where $K_{p}$ and $\sigma$ are two constants and $\varrho_{i+1 / 2}=\left(\varrho_{L}+\varrho_{R}\right) / 2$.

The mass flux at the interface $i+1 / 2$ is then written as:

$$
(\varrho v)_{i+1 / 2}=c_{i+1 / 2} M_{i+1 / 2} \varrho_{L}
$$

and the interface velocity $v_{i+1 / 2}^{\mathrm{T}}$ is given by the formula of $\mathrm{AUSM}^{+}$, Eq. (7), but now expressed with the interface Mach number given by Eq. (14). The numerical values of the parameters $K_{p}, K_{v}$ and $\sigma$ used in the subsequent test cases are those suggested in Ref. [8]: $K_{p}=0.25, K_{v}=0.75$ and $\sigma=1$. 
According to Eq. (15), the $\mathrm{AUSM}^{+}$-up contribution of the mass flux correction is written as

$$
(\varrho v)_{i+1 / 2, F}^{\prime}=c_{i+1 / 2}^{\star} M_{i+1 / 2}^{\prime} \varrho_{L}^{\star}
$$

where

$$
M_{i+1 / 2}^{\prime}=-\frac{K_{p}}{f\left(M_{0, i+1 / 2}^{\star}\right)} \max \left\{1-\left(\bar{M}_{i+1 / 2}^{\star}\right)^{2} \sigma, 0\right\} \frac{p_{i+1}^{\prime}-p_{i}^{\prime}}{\varrho_{i+1 / 2}^{\star}\left(c_{i+1 / 2}^{\star}\right)^{2}} .
$$

Thus, the pressure corrections are not extrapolated. This was found necessary in order to maintain the positivity of the correction system (13). Returning to Eq. (11), the dissipation coefficient arising from the $\mathrm{AUSM}^{+}$-up scheme is

$$
\kappa_{i+1 / 2}=\frac{K_{p}}{f\left(M_{0, i+1 / 2}^{\star}\right)} \max \left\{1-\left(\bar{M}_{i+1 / 2}^{\star}\right)^{2} \sigma, 0\right\} \frac{\varrho_{L}^{\star}}{\varrho_{i+1 / 2}^{\star} c_{i+1 / 2}^{\star}} .
$$

\subsection{Rhie-Chow interpolation alternatives}

The Rhie-Chow interpolation consists in the derivation of a transporting velocity from the momentum equation only, so that the contribution of a numerical flux function in Eq. (10) is excluded and $\kappa_{i+1 / 2}=0$ in Eq. (11). The procedure to derive an interpolated velocity is not unique. As illustrated further, a crucial issue is the time-step independency of the steady state. So, let us begin by the case of a steady flow before showing several alternatives of interpolation for unsteady flows.

\subsubsection{Steady flow}

In a steady flow, the momentum equation (2) can be written as:

$$
v_{i}^{\star \star}=\frac{B_{i}}{A_{i}}-\frac{1}{A_{i}}\left(p_{i+1 / 2}^{k}-p_{i-1 / 2}^{k}\right)
$$

where

$$
A_{i}=\varrho_{i}^{\star \star} v_{i+1 / 2}^{k} S_{i+1 / 2} / S_{i}
$$

and

$$
\begin{aligned}
B_{i}=-\frac{1}{S_{i}}[ & \frac{1}{2} \psi_{i}\left((\varrho v)^{k}\right)\left[(\varrho v)_{i}^{k}-(\varrho v)_{i-1}^{k}\right] v_{i+1 / 2}^{k} S_{i+1 / 2} \\
& \left.\left.-\left\{(\varrho v)_{i-1}^{k}+\frac{1}{2} \psi_{i-1}\left((\varrho v)^{k}\right)\left[(\varrho v)_{i-1}^{k}-(\varrho v)_{i-2}^{k}\right)\right]\right\} v_{i-1 / 2}^{k} S_{i-1 / 2}\right] .
\end{aligned}
$$


A relation at the cell-face similar to the one written at the cell-center in (16) is postulated:

$$
v_{i+1 / 2}^{T}=\frac{B_{i+1 / 2}}{A_{i+1 / 2}}-\frac{1}{A_{i+1 / 2}}\left(p_{i+1}^{k}-p_{i}^{k}\right)
$$

where the cell-face quantities in the RHS need to be specified. Then, a first option consists in the linear interpolation of $B$ independently of the interpolation of $A$, by setting:

$$
B_{i+1 / 2}=\frac{1}{2}\left(B_{i}+B_{i+1}\right)
$$

With this choice, the transporting velocity, in the form typically used in practice, is:

$$
\begin{aligned}
v_{i+1 / 2}^{\mathrm{T}} & =\frac{1}{2 A_{i+1 / 2}}\left(A_{i} v_{i}^{\star \star}+A_{i+1} v_{i+1}^{\star \star}\right) \\
+ & \frac{1}{A_{i+1 / 2}}\left[\frac{1}{2}\left(p_{i+3 / 2}^{k}-p_{i+1 / 2}^{k}\right)+\frac{1}{2}\left(p_{i+1 / 2}^{k}-p_{i-1 / 2}^{k}\right)-\left(p_{i+1}^{k}-p_{i}^{k}\right)\right] .
\end{aligned}
$$

A second option consists in the linear interpolation of the ratio $B / A$, namely:

$$
\left(\frac{B}{A}\right)_{i+1 / 2}=\frac{1}{2}\left[\left(\frac{B}{A}\right)_{i}+\left(\frac{B}{A}\right)_{i+1}\right]
$$

which leads to:

$$
\begin{aligned}
v_{i+1 / 2}^{\mathrm{T}}=\frac{1}{2}\left(v_{i}^{\star \star}+v_{i+1}^{\star \star}\right)+\frac{1}{2 A_{i+1}}\left(p_{i+3 / 2}^{k}-p_{i+1 / 2}^{k}\right) & +\frac{1}{2 A_{i}}\left(p_{i+1 / 2}^{k}-p_{i-1 / 2}^{k}\right) \\
& -\frac{1}{A_{i+1 / 2}}\left(p_{i+1}^{k}-p_{i}^{k}\right) .
\end{aligned}
$$

This latter option is sometimes referred as the original Rhie-Chow interpolation method [11]. In Eqs. (18) and (20), a common choice is:

$$
\frac{1}{A_{i+1 / 2}}=\frac{1}{2}\left(\frac{1}{A_{i}}+\frac{1}{A_{i+1}}\right) .
$$

The expression (18) was suggested by Lien and Leschziner [6] and by Pascau [11], but not used for steady flows. The expression (20) was used for steady flows by Miller and Schmidt [10], Majumdar [9] and Lien and Leschziner [6], to make the result independent of the underrelaxation coefficient. In case of underrelaxation of the velocity, both choices (17) and (19) lead to a transporting velocity independent of the underrelaxation coefficient at steady state. As recognized by Pascau [11], both choices ensure the time-step independency of the transporting velocity at steady state, when unsteady equations are considered. 


\subsubsection{Unsteady flow}

Keeping the notations of the steady case in Sec. 3.2.1, the momentum equation (2) with its inertial terms leads to

$$
B_{i}=A_{i} v_{i}^{\star \star}+\left(p_{i+1 / 2}^{k}-p_{i-1 / 2}^{k}\right)-\frac{2}{\tau}(\varrho v)_{i}^{n}+\frac{1}{2 \tau}(\varrho v)_{i}^{n-1}+\frac{3}{2 \tau}(\varrho v)_{i}^{\star \star}
$$

As previously in Sec. 3.2.1, a similar equation written at the cell-face is postulated:

$$
\begin{aligned}
B_{i+1 / 2}=A_{i+1 / 2} v_{i+1 / 2}^{T}+\left(p_{i+1}^{k}\right. & \left.-p_{i}^{k}\right)-\frac{2}{\tau} \varrho_{i+1 / 2}^{n}\left(v_{i+1 / 2}^{\mathrm{T}}\right)^{n} \\
& +\frac{1}{2 \tau} \varrho_{i+1 / 2}^{n-1}\left(v_{i+1 / 2}^{\mathrm{T}}\right)^{n-1}+\frac{3}{2 \tau} \varrho_{i+1 / 2}^{\star \star} v_{i+1 / 2}^{T} .
\end{aligned}
$$

It is worth to notice that the cell-face velocity has to be stored at each time-step. The option that corresponds to Eq. (17) leads to:

$$
\begin{aligned}
& v_{i+1 / 2}^{\mathrm{T}}=\frac{1}{2 A_{i+1 / 2}}\left(A_{i} v_{i}^{\star \star}+A_{i+1} v_{i+1}^{\star \star}\right) \\
& +\frac{1}{A_{i+1 / 2}}\left[\frac{1}{2}\left(p_{i+3 / 2}^{k}-p_{i+1 / 2}^{k}\right)+\frac{1}{2}\left(p_{i+1 / 2}^{k}-p_{i-1 / 2}^{k}\right)-\left(p_{i+1}^{k}-p_{i}^{k}\right)\right] \\
& +\frac{1}{A_{i+1 / 2}}\left[\frac{2}{\tau} \varrho_{i+1 / 2}^{n}\left(v_{i+1 / 2}^{\mathrm{T}}\right)^{n}-\frac{1}{2 \tau} \varrho_{i+1 / 2}^{n-1}\left(v_{i+1 / 2}^{\mathrm{T}}\right)^{n-1}-\frac{3}{2 \tau} \varrho_{i+1 / 2}^{\star \star} v_{i+1 / 2}^{\mathrm{T}}\right] \\
& -\frac{1}{2 A_{i+1 / 2}}\left[\frac{2}{\tau}(\varrho v)_{i}^{n}-\frac{1}{2 \tau}(\varrho v)_{i}^{n-1}-\frac{3}{2 \tau}(\varrho v)_{i}^{\star \star}\right. \\
& \left.\quad+\frac{2}{\tau}(\varrho v)_{i+1}^{n}-\frac{1}{2 \tau}(\varrho v)_{i+1}^{n-1}-\frac{3}{2 \tau}(\varrho v)_{i+1}^{\star \star}\right]
\end{aligned}
$$

With form (23), where $v^{\mathrm{T}}$ is present in both sides of the equation, it is clear that the expression (18) in the steady case is recovered if a steady state is reached, so that this steady state is independent of the time-step. Another important feature of both expressions (22) and (23) (presented in Ref. [6] and used e.g. in Ref. [20]) is that the dependency of the face velocity $v_{i+1 / 2}^{\mathrm{T}}$ on the pressure difference involves a coefficient that contains explicitly the time-step.

The option that corresponds to Eq. (19) is suggested in Ref. [11]. It leads to an expression of $v^{\mathrm{T}}$ which also satisfies the two properties just mentioned. In this 
case, the transporting velocity reads:

$$
\begin{aligned}
& v_{i+1 / 2}^{\mathrm{T}}=\frac{1}{2 a_{i+1 / 2}}\left(v_{i}^{\star \star}+v_{i+1}^{\star \star}\right) \\
& +\frac{1}{a_{i+1 / 2}}\left[\frac{1}{2 A_{i+1}}\left(p_{i+3 / 2}^{k}-p_{i+1 / 2}^{k}\right)+\frac{1}{2 A_{i}}\left(p_{i+1 / 2}^{k}-p_{i-1 / 2}^{k}\right)-\frac{1}{A_{i+1 / 2}}\left(p_{i+1}^{k}-p_{i}^{k}\right)\right] \\
& +\frac{1}{a_{i+1 / 2}}\left\{\frac{2}{\tau}\left[\frac{1}{A_{i+1 / 2}} \varrho_{i+1 / 2}^{n}\left(v_{i+1 / 2}^{\mathrm{T}}\right)^{n}-\frac{1}{2 A_{i}}(\varrho v)_{i}^{n}-\frac{1}{2 A_{i+1}}(\varrho v)_{i+1}^{n}\right]\right. \\
& -\frac{1}{2 \tau}\left[\frac{1}{A_{i+1 / 2}} \varrho_{i+1 / 2}^{n-1}\left(v_{i+1 / 2}^{\mathrm{T}}\right)^{n-1}-\frac{1}{2 A_{i}}(\varrho v)_{i}^{n-1}-\frac{1}{2 A_{i+1}}(\varrho v)_{i+1}^{n-1}\right] \\
& \left.+\frac{3}{2 \tau}\left[\frac{1}{2 A_{i}}(\varrho v)_{i}^{\star \star}+\frac{1}{2 A_{i+1}}(\varrho v)_{i+1}^{\star \star}\right]\right\}
\end{aligned}
$$

where

$$
a_{i+1 / 2}=1+\frac{1}{A_{i+1 / 2}} \frac{3}{2 \tau} \varrho_{i+1 / 2}^{\star \star} .
$$

In Ref. [15], a variant is suggested to avoid non physical oscillations in the solution when small time-steps are used. In fact, this variant was already suggested in Ref. [2]. In this case, Eq. (21) is rewritten as:

$$
\alpha_{i} v_{i}^{\star \star}=B_{i}-\left(p_{i+1 / 2}^{k}-p_{i-1 / 2}^{k}\right)+\frac{2}{\tau}(\varrho v)_{i}^{n}-\frac{1}{2 \tau}(\varrho v)_{i}^{n-1}
$$

where

$$
\alpha_{i}=A_{i}+\frac{3}{2 \tau} \varrho_{i}^{\star \star}
$$

Linear interpolation of the ratio $B / \alpha$ yields:

$$
\begin{aligned}
& v_{i+1 / 2}^{\mathrm{T}}=\frac{1}{2}\left(v_{i}^{\star \star}+v_{i+1}^{\star \star}\right) \\
& +\left[\frac{1}{2 \alpha_{i}}\left(p_{i+1 / 2}^{k}-p_{i-1 / 2}^{k}\right)+\frac{1}{2 \alpha_{i+1}}\left(p_{i+3 / 2}^{k}-p_{i+1 / 2}^{k}\right)-\frac{1}{\alpha_{i+1 / 2}}\left(p_{i+1}^{k}-p_{i}^{k}\right)\right] \\
& -\frac{2}{\tau}\left[\frac{1}{2 \alpha_{i}}(\varrho v)_{i}^{n}+\frac{1}{2 \alpha_{i+1}}(\varrho v)_{i+1}^{n}-\frac{1}{\alpha_{i+1 / 2}} \varrho_{i+1 / 2}^{n}\left(v_{i+1 / 2}^{\mathrm{T}}\right)^{n}\right] \\
& \quad+\frac{1}{2 \tau}\left[\frac{1}{2 \alpha_{i}}(\varrho v)_{i}^{n-1}+\frac{1}{2 \alpha_{i+1}}(\varrho v)_{i+1}^{n-1}-\frac{1}{\alpha_{i+1 / 2}} \varrho_{i+1 / 2}^{n-1}\left(v_{i+1 / 2}^{\mathrm{T}}\right)^{n-1}\right]
\end{aligned}
$$

where

$$
\frac{1}{\alpha_{i+1 / 2}}=\frac{1}{2}\left(\frac{1}{\alpha_{i}}+\frac{1}{\alpha_{i+1}}\right) .
$$

Obviously in this case, the steady state, if it exists, is time-step dependent. 
Table 1

Scalings of the transporting velocity coefficients for low Mach number acoustic problems

\begin{tabular}{llllc}
\hline & Convection term & Pressure term & Inertia term & Time consistency \\
\hline Refs. [6,11] : & $\left(1+\frac{1}{\tau \mathrm{M}}\right)^{-1}$ & $\left(\mathrm{M}^{2}+\frac{\mathrm{M}}{\tau}\right)^{-1}$ & $(1+\tau \mathrm{M})^{-1}$ & yes \\
\hline Refs. [2,15] : & $\left(1+\frac{1}{\tau \mathrm{M}}\right)^{-1}$ & $\left(\mathrm{M}^{2}+\frac{\mathrm{M}}{\tau}\right)^{-1}$ & $(1+\tau \mathrm{M})^{-1}$ & no \\
\hline
\end{tabular}

\subsubsection{Low Mach number aspects}

It is important to check if the transporting velocities considered in section 3.2.2 preserve the low Mach number scaling of the discretized momentum equation (21). Let us introduce a reference length $l_{\mathrm{r}}$, pressure $p_{\mathrm{r}}$, density $\varrho_{\mathrm{r}}$, velocity $v_{\mathrm{r}}$ and time $t_{\mathrm{r}}$. We choose the length of the flow domain as reference length. Further reference values are the pressure, density and convective velocity $v_{\mathrm{r}}$ of the background low Mach number flow. We consider the velocity $\sqrt{p_{\mathrm{r}} / \varrho_{\mathrm{r}}}$, which is of the same order as the velocity of sound in the mean flow. The reference Mach number is thus defined as $\mathrm{M}=v_{\mathrm{r}} / \sqrt{p_{\mathrm{r}} / \varrho_{\mathrm{r}}}$. Our purpose is to study acoustic propagation in low Mach number flow. Therefore, the reference time is of the order of the time needed by an acoustic wave to travel over the reference length, thus $t_{\mathrm{r}}=l_{\mathrm{r}} / \sqrt{p_{\mathrm{r}} / \varrho_{\mathrm{r}}}$. The non-dimensional form of the discretized momentum equation (21) becomes

$$
B_{i}=A_{i} v_{i}^{\star \star}+\frac{1}{\mathrm{M}^{2}}\left(p_{i+1 / 2}^{k}-p_{i-1 / 2}^{k}\right)-\frac{2 \mathrm{~S}_{\mathrm{t}}}{\tau}(\varrho v)_{i}^{n}+\frac{\mathrm{S}_{\mathrm{t}}}{2 \tau}(\varrho v)_{i}^{n-1}+\frac{3 \mathrm{~S}_{\mathrm{t}}}{2 \tau}(\varrho v)_{i}^{\star \star}
$$

where $\mathrm{S}_{\mathrm{t}}=\left(l_{\mathrm{r}} / v_{\mathrm{r}}\right) / t_{\mathrm{r}}$ is the reference Strouhal number. The Mach number scaling of the transporting velocities considered in section 3.2.2 proves to be identical. As an illustration, the use of the linear interpolation given in (17) (cf. Lien and Leschziner, 1994 [6]) leads to the following expression of the transporting velocity:

$$
\begin{aligned}
v_{i+1 / 2}^{\mathrm{T}}=K_{C} B_{i+1 / 2}- & K_{P}\left(p_{i+1}^{k}-p_{i}^{k}\right) \\
& +K_{I}\left[2 \varrho_{i+1 / 2}^{n}\left(v_{i+1 / 2}^{\mathrm{T}}\right)^{n}-\frac{1}{2} \varrho_{i+1 / 2}^{n-1}\left(v_{i+1 / 2}^{\mathrm{T}}\right)^{n-1}\right]
\end{aligned}
$$

where

$$
K_{C}=\left(A_{i+1 / 2}+\frac{3 \mathrm{~S}_{\mathrm{t}}}{2 \tau} \varrho_{i+1 / 2}^{\star \star}\right)^{-1} \quad, \quad K_{P}=\frac{K_{C}}{\mathrm{M}^{2}} \quad, \quad K_{I}=K_{C} \frac{\mathrm{S}_{\mathrm{t}}}{\tau}
$$

are the coefficients of the convection, pressure and inertia terms. For acoustic problems in low Mach number flow, the reference time is $t_{\mathrm{r}}=\mathrm{M} l_{\mathrm{r}} / v_{\mathrm{r}}$ (cf. e.g. Ref. [19]), so that the reference Strouhal number $\mathrm{S}_{\mathrm{t}}$ belongs to $\mathcal{O}(1 / \mathrm{M})$. Thus, the scaling behaviour of coefficients (28) can be expressed in terms of $\tau$ and M only. This corresponds to the scaling given term by term in Table 1 . The time consistency property refers to the ability of the methods to obtain a steady state independent of the time-step. 
The previous non-dimensional formulation evidences that the Mach number scaling properties of the discretized momentum equation (21) are preserved by the transporting velocities considered in section 3.2.2. Therefore, these Mach number scaling properties will be referred to as the proper ones.

The pressure-velocity coupling coefficient, which is the pressure term in Table 1, second column, consists in a steady scaling term in $\mathrm{M}^{2}$ and an unsteady scaling term in $\mathrm{M} / \tau$. The steady scaling must be recovered when a steady state is reached after a transient phase, that is, as soon as we can take $\Delta t=\tau \Delta x \rightarrow+\infty$. Let us emphasize that this condition is immediately satisfied with Momentum Interpolation methods.

For the $\mathrm{AUSM}^{+}$-up scheme, the proper steady scaling is obtained through the scaling function $f$, such that $f(\mathrm{M}) \in \mathcal{O}(\mathrm{M})$ when $\mathrm{M} \searrow 0$ (see Eq. (4)). Notice that the steady scaling is solely accounted for by the $\mathrm{AUSM}^{+}$-up scheme, which is derived from an analysis of steady equations. However, for acoustic simulation in low Mach number flow, it is demonstrated by Dellacherie [3] that the pressure-velocity coupling in the $\mathrm{AUSM}^{+}$-up scheme is suitable to avoid spurious acoustic waves, and this is not the case, unless $\Delta x \in \mathcal{O}(\mathrm{M})$, for the AUSM or AUSM ${ }^{+}$schemes (see [8]), which do not involve the proper steady scaling. Therefore, for acoustic simulations in low Mach number flow, we shall consider the $\mathrm{AUSM}^{+}$-up scheme, with the pressure-velocity coupling that involves the scaling function given in Eq. (4).

The comparison between the $\mathrm{AUSM}^{+}$-up scheme and the Momentum Interpolation methods will reveal the role of the unsteady low Mach number scaling. As the scaling properties are the same for all the Momentum Interpolation methods considered in this study, the comparison of the methods on unsteady numerical experiments will make clear the practical importance of the time consistency property.

\section{Numerical experiments}

A series of numerical test cases is performed with the intention to investigate a suitable choice of the transporting velocity allowing unsteady calculations, with the possibility of acoustic features. Practically, five iterations per time-step are sufficient.

\subsection{Boundary treatment}

A characteristic-based treatment is applied to the boundary, following Thompson [17] and Poinsot and Lele [12]. The primitive variables $\varrho, v$ and $p$ are calculated at 
the inlet and the outlet by solving the LODI (for Locally One Dimensional Inviscid) equations, written as

$$
\begin{aligned}
& \partial_{t} \varrho+\frac{\varrho}{2 c}\left(\mathcal{L}_{1}+\mathcal{L}_{3}\right)+\mathcal{L}_{2}=-\varrho v \frac{\mathrm{d}_{x} S}{S} \\
& \partial_{t} v+\frac{1}{2}\left(\mathcal{L}_{3}-\mathcal{L}_{1}\right)=0 \\
& \partial_{t} p+\frac{\varrho c}{2}\left(\mathcal{L}_{1}+\mathcal{L}_{3}\right)=-\varrho v c^{2} \frac{\mathrm{d}_{x} S}{S}
\end{aligned}
$$

where the quantities

$$
\begin{aligned}
\mathcal{L}_{1} & =(v-c)\left(\frac{1}{\varrho c} \partial_{x} p-\partial_{x} v\right) \\
\mathcal{L}_{2} & =v\left(\partial_{x} \varrho-\frac{1}{c^{2}} \partial_{x} p\right) \\
\mathcal{L}_{3} & =(v+c)\left(\frac{1}{\varrho c} \partial_{x} p+\partial_{x} v\right) .
\end{aligned}
$$

are interpreted as the temporal rate of change of the wave amplitudes [12]. At the outlet, the amplitude of the incoming acoustic wave is written as

$$
\mathcal{L}_{1}=k_{p}\left(p-p^{\dagger}\right)
$$

where the superscript $\dagger$ denotes a target value. At the inlet, the amplitudes of the incoming convective and acoustic waves are written with the same convention as

$$
\mathcal{L}_{3}=k_{v}\left(v-v^{\dagger}\right) \quad, \quad \mathcal{L}_{2}=k_{\varrho}\left(\varrho-\varrho^{\dagger}\right)
$$

The filtering level of the boundary is tuned through the relaxation coefficients $k_{p}$, $k_{v}$ and $k_{\varrho}$, following Selle et al. [14].

\subsection{Steady low Mach number flow}

As a preliminary test, before considering numerical experiments involving an unsteady flow and acoustic features, let us check the ability of the algorithm described in Sec. 2 to calculate steady low Mach number solutions, when the transporting velocity is one of the four given in Sec. 3. For this test, a one-meter long convergingdiverging nozzle is considered. Its section (dimensions in meter) is given by

$$
S(x)=\left\{\begin{array}{lr}
0.1, & 0 \leq x \leq 2 / 28 \\
0.1\left\{0.9+0.1\left[2\left(\frac{x-\frac{11}{28}}{\frac{9}{28}}\right)^{2}-\left(\frac{x-\frac{11}{28}}{\frac{9}{28}}\right)^{4}\right]\right\}, 2 / 28 \leq x \leq 20 / 28 \\
0.1, & 20 / 28 \leq x \leq 1
\end{array}\right.
$$


The fluid is air. The initial conditions are:

$$
\left\{\begin{array}{l}
\varrho^{0}=1.2046 \mathrm{~kg} \mathrm{~m}^{-3} \\
v^{0}=0 \\
p^{0}=101300 \mathrm{~Pa}
\end{array}\right.
$$

The boundary conditions are given by (see Eqs. (29)-(30)):

$$
\varrho^{\dagger}=1.2046 \mathrm{~kg} \mathrm{~m}^{-3}, \quad v^{\dagger}=0.30886 \mathrm{~m} \mathrm{~s}^{-1}, \quad p^{\dagger}=101300 \mathrm{~Pa}
$$

At convergence, the Mach number at the throat of the nozzle is $10^{-3}$ and is around $910^{-4}$ at the inlet and the outlet of the nozzle, where the section is constant. This latter value is chosen for the cut-off Mach number $M_{\text {co }}$ in Eq. (3) and the AUSM ${ }^{+}$ up construction described in Sec. 3.1. The convective CFL number is 0.1. Thus, the acoustic CFL number is around 100. The axis of the nozzle is divided into 100 cells with a constant mesh spacing $\Delta x$. For this test only, the MinMod slope limiter is used. The Bounded Central limiter is used elsewhere.

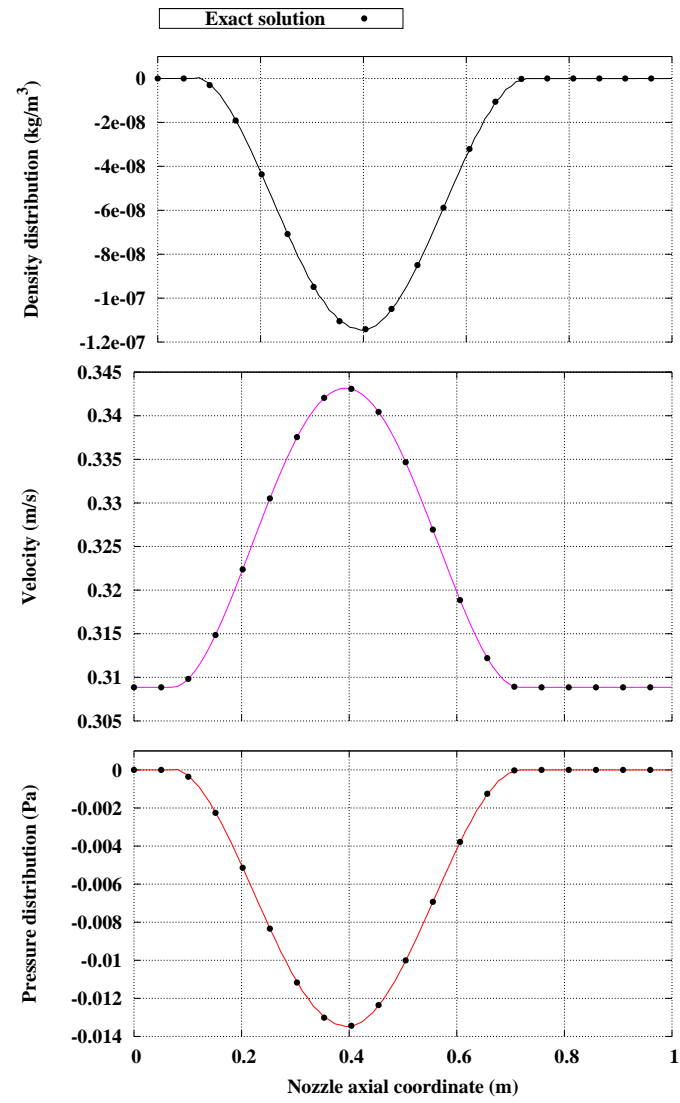

(a) Eqs. (7) and (14), $c f$. Liou, 2006 [8].

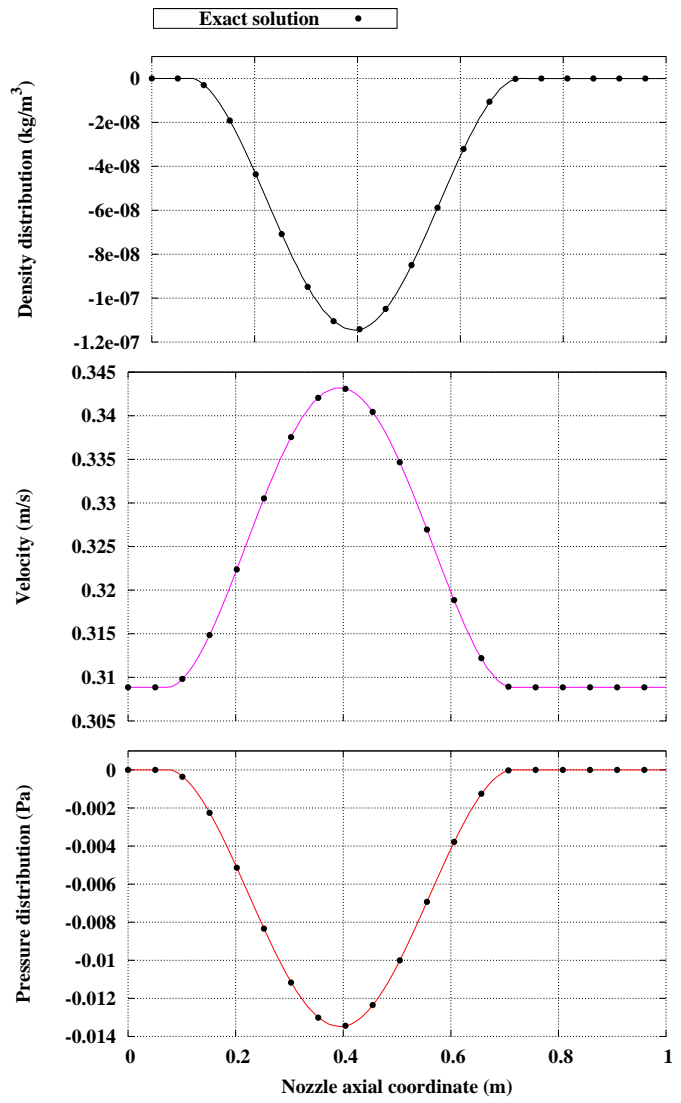

(b) Eq. (23), cf. Lien et al., 1994 [6].

Fig. 1. Steady low Mach number flow through a converging/diverging nozzle.

The solutions are shown in Figs. 1-2 (see e.g. Ref. [1] for the exact solution). As 


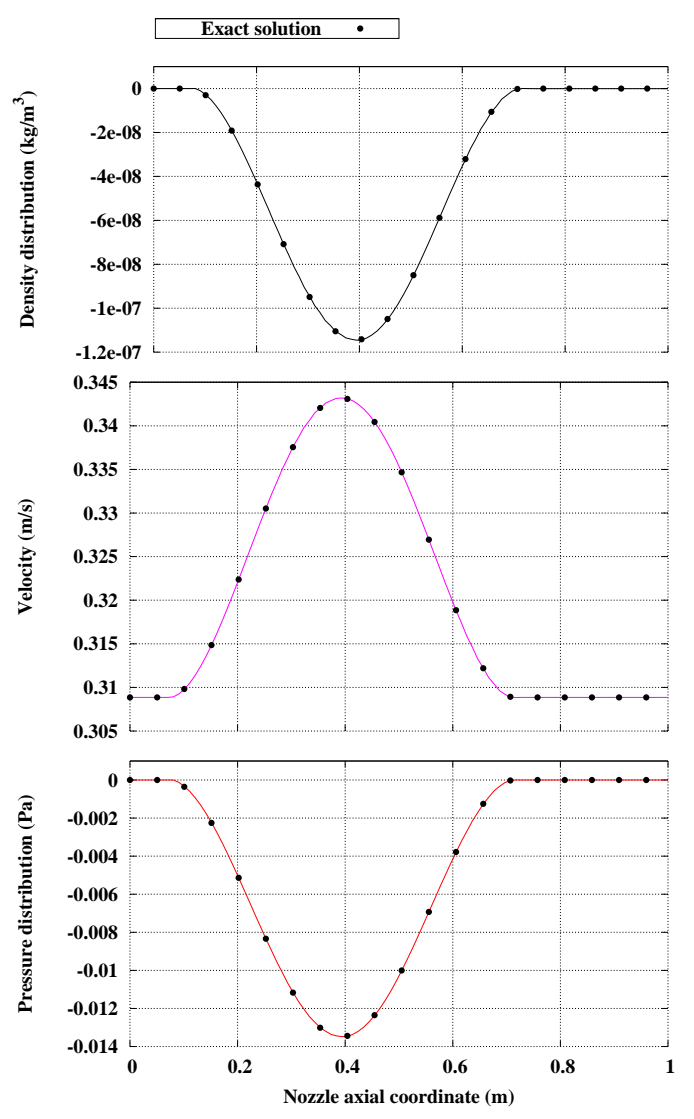

(a) Eq. (24), $c f$. Pascau, 2011 [11].

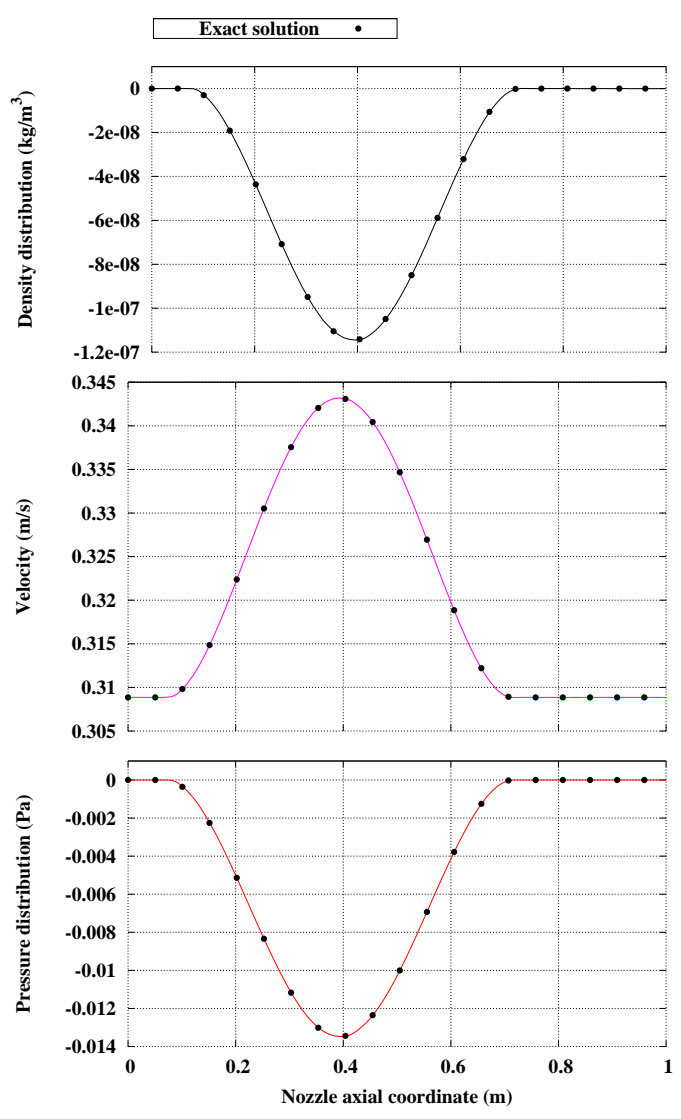

(b) Eq. (25), cf. Shen et al., 2001 [15].

Fig. 2. Steady low Mach number flow.

expected, the pressure correction method of Sec. 2 combined with the various transporting velocities of Sec. 3 gives results free of checkerboard decoupling oscillations. All variants of the face velocity expressions have the ability of calculating accurately the steady low Mach number flow solutions.

\subsection{Unsteady low Mach number flows}

A series of distinctive unsteady test cases is investigated in this section. We first consider a low Mach number flow in which unsteadiness is achieved through a time-varying inlet velocity. For this first unsteady test, the frequency of the sollicitation is kept constant. Secondly, the advection of an acoustic pulse is addressed. The choice of a narrow signal, that possesses a broad spectrum in space, is suitable to reveal the dispersive features of a scheme (see e.g. Ref. [18]). Finally, the interference of two acoustic pulses is considered, as an illustration of the capability of the method selected from the previous test cases. In each test case, the data are chosen in order to obtain linear acoustic features, allowing a direct comparison with the exact solution. 


\subsubsection{Inlet velocity oscillation}

To evidence the suitability of a definition of the face velocity with time-step dependency of the pressure-velocity coupling, the propagation of a wave forced at the left boundary of a nozzle with constant section (a pipe) is now considered. The pipe is one-meter long and divided into 500 cells. The cut-off Mach number $\mathrm{M}_{\mathrm{co}}$ is $910^{-4}$. The initial conditions are:

$$
\left\{\begin{array}{l}
\varrho^{0}=1.2046 \mathrm{~kg} \mathrm{~m}^{-3} \\
v^{0}=0.30886 \mathrm{~m} \mathrm{~s}^{-1} \\
p^{0}=101300 \mathrm{~Pa}
\end{array}\right.
$$

The simulation length is chosen such that no wave reaches the outlet before the time $t_{f}=2 \mathrm{~ms}$. The inlet velocity is specified to oscillate about a mean value $V$, as

$$
v^{\dagger}(t)=V[1+A \sin (2 \pi f t)]
$$

where $V=0.30886 \mathrm{~m} \mathrm{~s}^{-1}, A=10^{-2}$ and $f=210^{3} \mathrm{~Hz}$. The other target values required at the boundary are: $\varrho^{\dagger}=1.2046 \mathrm{~kg} \mathrm{~m}^{-3}$ and $p^{\dagger}=101300 \mathrm{~Pa}$. The convective CFL number is chosen as $10^{-4}$. With this value, the acoustic CFL number is around 0.1 in the domain reached by the wave. Thus, the acoustic waves generated through the sinusoidal variations of the inlet velocity, can be accurately calculated.

Results at $t_{f}=2 \mathrm{~ms}$ shown in Fig. 3 demonstrate that the transporting velocity given through the Rhie-Chow interpolation in Eqs. (23) (Lien et al., 1994), (24) (Pascau, 2011) and (25) (Shen et al., 2001) are very close to each other. As expected, these methods properly take into account the inlet oscillations. Moreover, the velocity of the wave front propagation is very close to the one obtained from the linear acoustic theory. Acoustics is involved since the oscillating inlet velocity corresponds to the oscillation of the incoming wave amplitude $\mathcal{L}_{3}$ (see Eq. (30)). Finally, the density, velocity and pressure profiles obtained when $\mathrm{AUSM}^{+}$-up interpolation is used do not reproduce correctly the oscillations (see Fig. 3, bottom). The pressure-velocity coupling of this scheme is not time-step dependent and therefore, can not capture the variations imposed at the inlet, despite the small time-step chosen. Moreover, the position of the wave front is not well calculated. Thus, the inadequation of the $\mathrm{AUSM}^{+}$-up interpolation to accurately calculate acoustic waves in a low Mach number flow is evidenced. This scheme will, therefore, not be considered in the following numerical experiments.

\subsubsection{Acoustic pulse propagation}

A narrow acoustic pulse is generated through an initial acoustic Gaussian perturbation superimposed onto a mean flow, with constant density $\varrho_{0}$, velocity $v_{0}$ and 

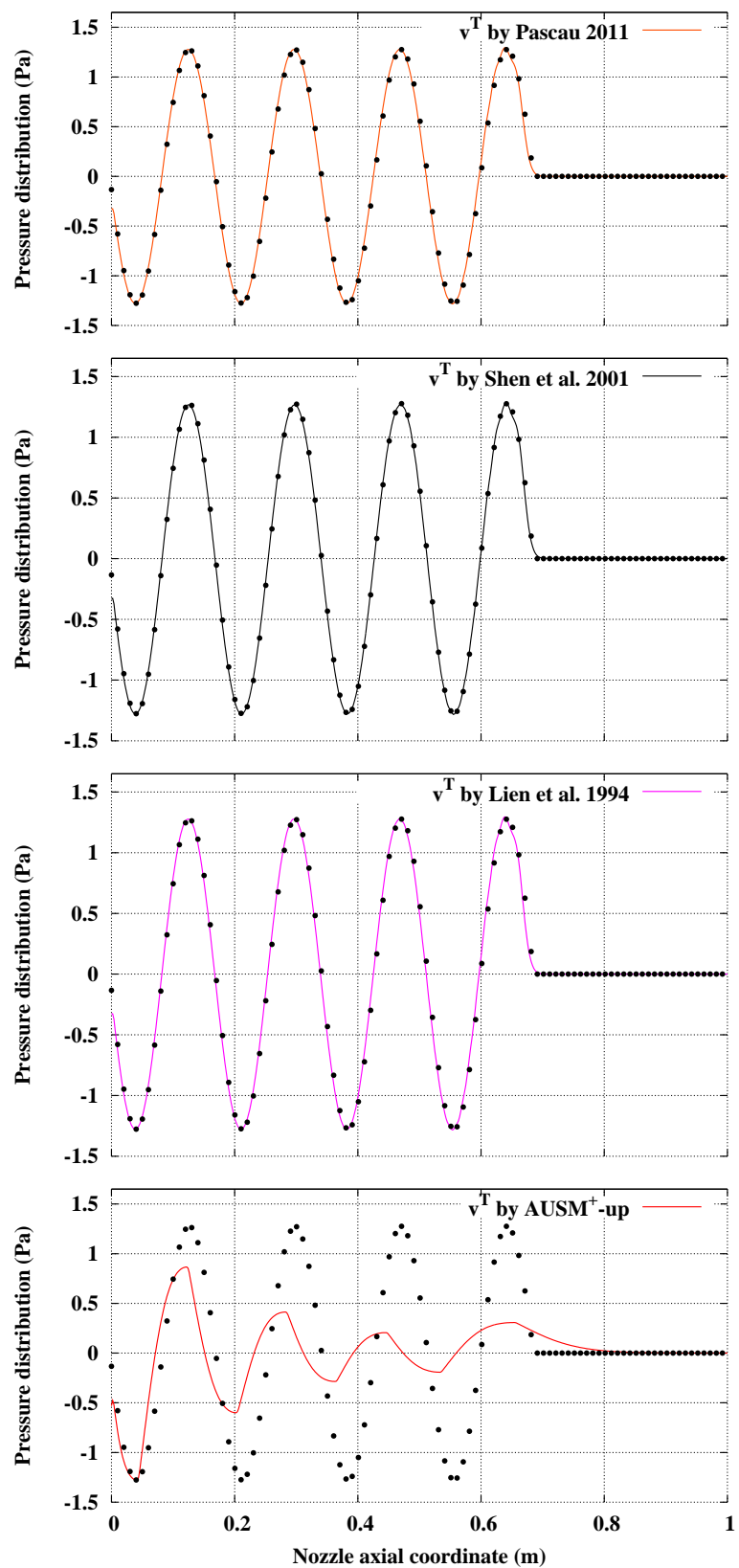

Fig. 3. Propagation of a wave forced at the left boundary. Pressure distribution, $t=210^{-3} \mathrm{~s}$. Exact solution (linear acoustic theory):

pressure $p_{0}$, in a five-meter long pipe. Thus, $p=p_{0}+\delta p$, where at $t=0$,

$$
\delta p=A \exp \left(-\frac{(x-m)^{2}}{2 s^{2}}\right) \text {. }
$$

By taking $\delta v=\delta p /\left(\varrho_{0} c_{0}\right)$ and $\delta \varrho=\delta p / c_{0}^{2}$, a pulse that propagates to the right (downstream) is obtained. In this case, the initial position is given by $m=0.2 \mathrm{~m}$. To obtain a pulse travelling to the left (upstream), initial values are such that: $\delta v=$ $-\delta p /\left(\varrho_{0} c_{0}\right)$ and $\delta \varrho=\delta p / c_{0}^{2}$. In this case $m=4.8 \mathrm{~m}$. The dimensional numerical settings for the wave generation are given in Table 2 . 


\begin{tabular}{|c|c|c|c|c|c|}
\hline$A(\mathrm{~Pa})$ & $s(\mathrm{~m})$ & $m(\mathrm{~m})$ & $p_{0}(\mathrm{~Pa})$ & $v_{0}(\mathrm{~m} / \mathrm{s})$ & $\varrho_{0}\left(\mathrm{~kg} / \mathrm{m}^{3}\right)$ \\
\hline 200 & 2 & 0.2 or 4.8 & 101300 & 0.30886 & 1.2046 \\
\hline
\end{tabular}

Table 2

Settings for the acoustic pulse generation.

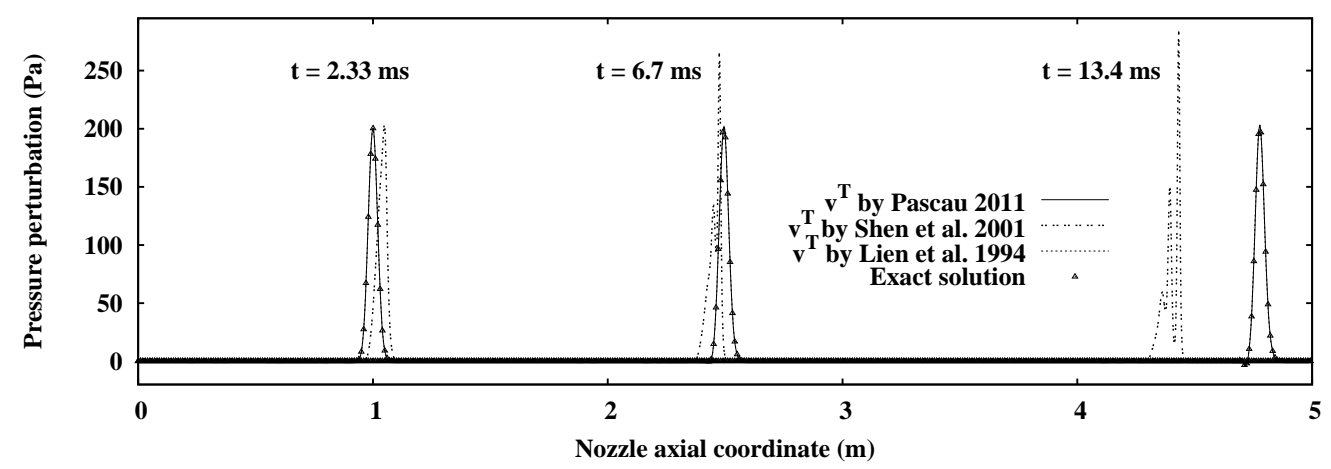

Fig. 4. Acoustic pulse propagation - Downstream propagation. Pressure distribution (results using Lien and Leschziner [6] and Pascau [11] formulations coincide).

A pipe divided into $N=2500$ cells is considered. The boundary conditions are those given by (32). The Mach number of the mean flow is $910^{-4}$, which is also the value chosen for $\mathrm{M}_{\mathrm{co}}$, and the convective CFL number is $510^{-5}$ (except in Fig. 8 where several values are compared). The acoustic CFL number is around 0.055.

The downstream propagation of the pulse is shown in Fig. 4. It is observed that its advection velocity is basically correct when the transporting velocities used are those given in Eqs. (23) (Lien et al., 1994) and (24) (Pascau, 2011). Moreover, the shape of the Gaussian pulse is conserved. On the contrary, using the transporting velocity given in Eq. (25) (Shen et al., 2001) leads to a large error, both in position and shape. In case of upstream propagation of the acoustic pulse, a glance at the Fig. 5 suffices to demonstrate that only the transporting velocities given by (23) (Lien et al., 1994) and (24) (Pascau, 2011) give satisfying results concerning the amplitude and position of the pulse.

In order to quantify the errors associated to the different choices of the transporting velocities, we follow here the procedure introduced by Takacs [16]. Say that $q_{e}$ and $q_{c}$ are exact and computed values of a quantity $q$ under consideration. At a given time $t$, the mean square error can then be defined as:

$$
\mathcal{E}^{2}=\frac{1}{N} \sum\left(q_{e}-q_{c}\right)^{2}
$$

where the sum extends over the $N$ nodes of the grid. Further, we define mean values 
and variances of the quantities by

$$
\begin{aligned}
& \bar{q}_{e}=\frac{1}{N} \sum q_{e}, \quad \bar{q}_{c}=\frac{1}{N} \sum q_{c} \\
& \sigma_{e}^{2}=\frac{1}{N} \sum\left(q_{e}-\bar{q}_{e}\right)^{2} \quad, \quad \sigma_{c}^{2}=\frac{1}{N} \sum\left(q_{c}-\bar{q}_{c}\right)^{2} .
\end{aligned}
$$

Then, the mean square error can be written as

$$
\begin{aligned}
\mathcal{E}^{2} & =\frac{1}{N} \sum\left[\left(q_{e}-\bar{q}_{e}\right)-\left(q_{c}-\bar{q}_{c}\right)+\left(\bar{q}_{e}-\bar{q}_{c}\right)\right]^{2} \\
& =\frac{1}{N} \sum\left(q_{e}-\bar{q}_{e}\right)^{2}+\frac{1}{N} \sum\left(q_{c}-\bar{q}_{c}\right)^{2}+\left(\bar{q}_{e}-\bar{q}_{c}\right)^{2}-\frac{2}{N} \sum\left(q_{e}-\bar{q}_{e}\right)\left(q_{c}-\bar{q}_{c}\right) \\
& =\sigma_{e}^{2}+\sigma_{c}^{2}+\left(\bar{q}_{e}-\bar{q}_{c}\right)^{2}-2 \operatorname{cov}\left(q_{e}, q_{c}\right)
\end{aligned}
$$

where $\operatorname{cov}\left(q_{e}, q_{c}\right)$ is the covariance of the two signals. The correlation coefficient between the two signals is then

$$
R\left(q_{e}, q_{c}\right)=\frac{\operatorname{cov}\left(q_{e}, q_{c}\right)}{\sigma_{e} \sigma_{c}} .
$$

The error (33) can further be written as

$$
\mathcal{E}^{2}=\left(\bar{q}_{e}-\bar{q}_{c}\right)^{2}+\left(\sigma_{e}-\sigma_{c}\right)^{2}+2 \sigma_{e} \sigma_{c}\left[1-R\left(q_{e}, q_{c}\right)\right]
$$

The different error components can now be recognized. The difference between $\bar{q}_{e}$ and $\bar{q}_{c}$ is the conservation error as the mean values of the signals express the content of the signals. With the conservative discretization used here, this error is zero. The difference between $\sigma_{e}$ and $\sigma_{c}$ is the dissipation error as the variances express the energy of the signals with respect to their mean values. The remaining component was considered by Takacs [16] as the dispersion error, as for exact correlation between the signals, the only error that can occur is due to dissipation. This way of denoting the error does not conform completely with the now commonly used concept of the modified wave number to express dissipation and dispersion errors. In order to see the relation with the modified wave number concept, a Fourier component may be substituted into (34). This then reveals (not derived here) the rather obvious result that non-dimensional measures of dissipation and dispersion errors may be defined by

$$
\hat{\mathcal{E}}_{\text {dissipation }}=\frac{\sigma_{e}-\sigma_{c}}{\sigma_{e}}, \hat{\mathcal{E}}_{\text {dispersion }}=\sqrt{1-R\left(q_{e}, q_{c}\right)} .
$$

For exact solutions, the error measures are zero. The error measure for dissipation becomes unity when all energy dissipates away in the computational result. The error measure for dispersion becomes unity when the correlation between exact and computational solutions disappears completely. The error analysis with (35) of the results shown in figures 4 and 5 reveals that the dispersion error dominates, which 


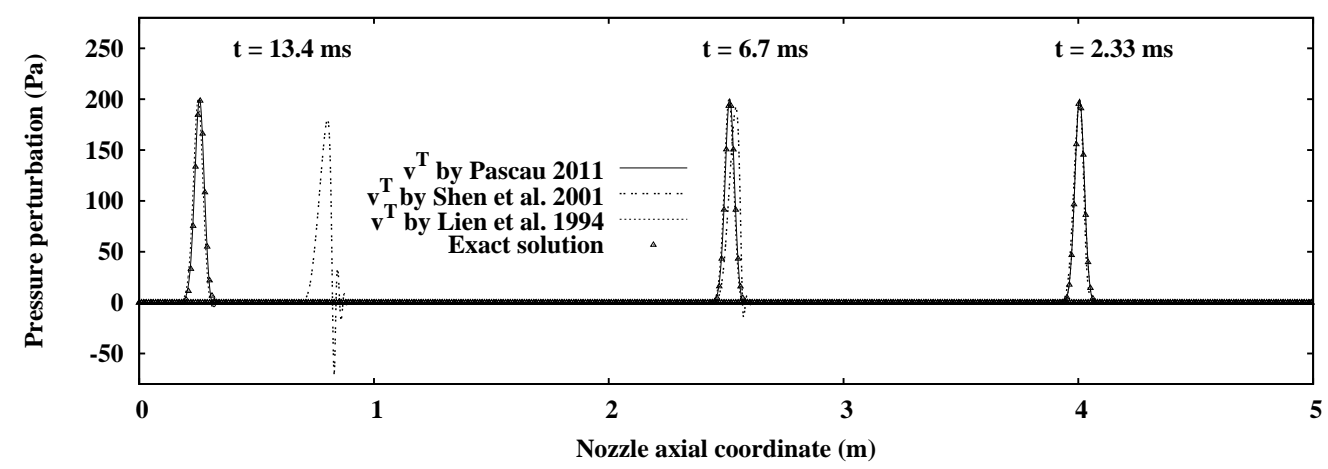

Fig. 5. Acoustic pulse propagation - Upstream propagation. Pressure distribution (results using Lien and Leschziner [6] and Pascau [11] formulations coincide).

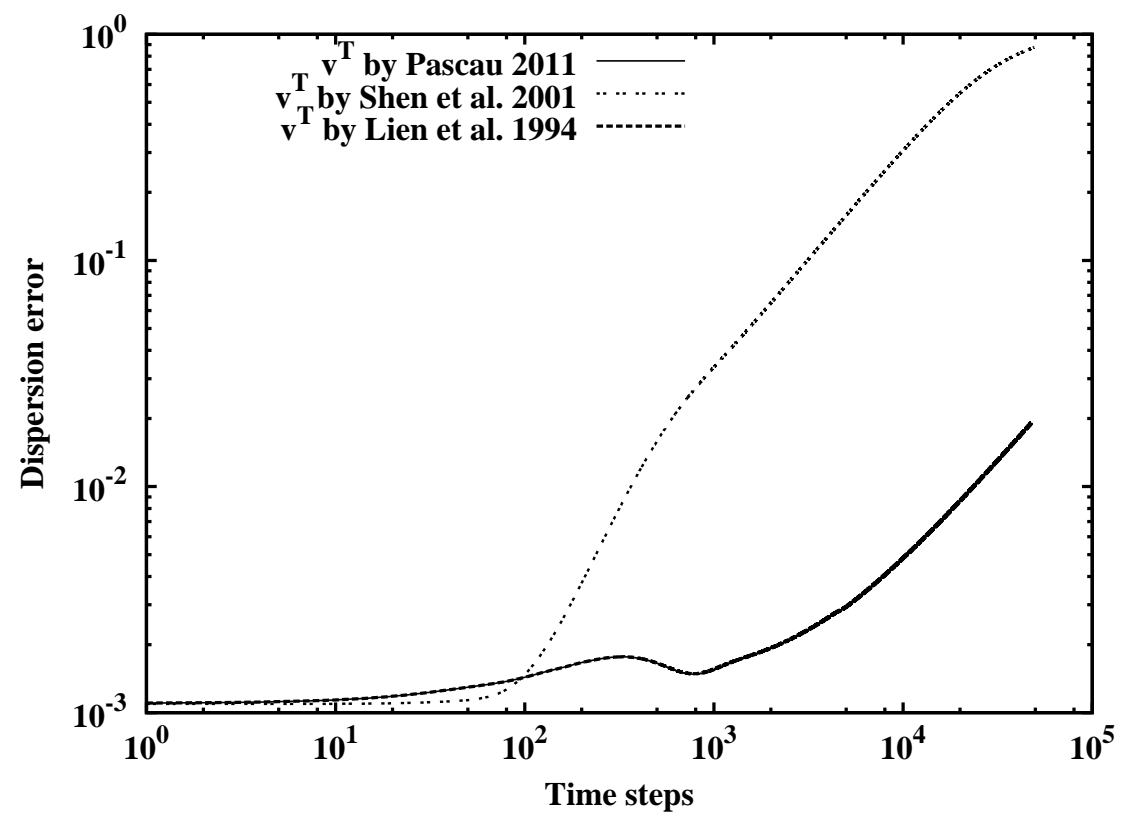

Fig. 6. Acoustic pulse propagation - Downstream propagation. Dispersion error vs. time-steps (results using Lien and Leschziner [6] and Pascau [11] formulations coincide).

is already obvious when inspecting the figures. The evolution of the dispersion errors in shown in figures 6 and 7. For a correct interpretation of the results, we should keep in mind that the dispersion error in the time domain is a quantity that grows with time according to (35). The nowadays common modified wave number analysis, on the other hand, produces error factors per time step (so not for the evolved time in the computation), that depend on the wave number. In figures 6 and 7 , it is clearly demonstrated that the dispersion errors with the transporting velocity given by (25) (Shen et al., 2001) are extremely large.

Suppose that, based on the above considerations, an adequate transporting velocity is chosen, for instance the one given in Eq. (24) (Pascau, 2011). Then, the simulation can still be useless, unless a carreful choice of the CFL parameter has been 


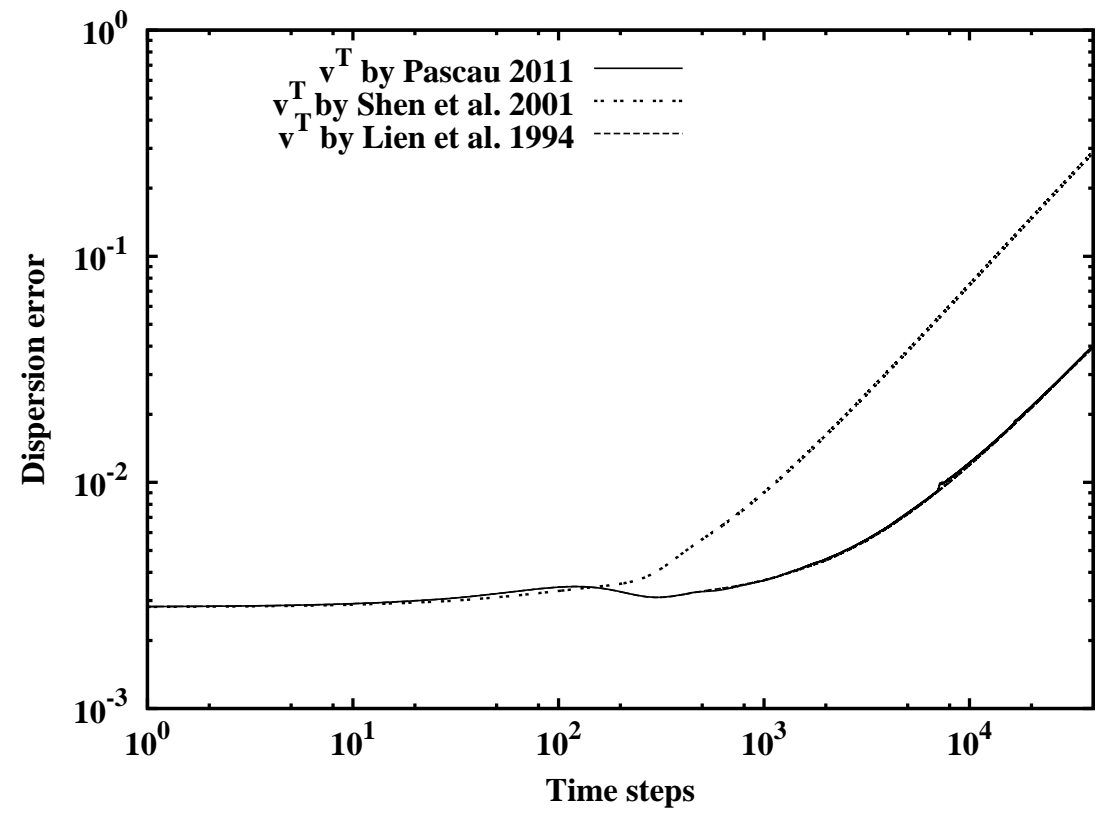

Fig. 7. Acoustic pulse propagation - Upstream propagation. Dispersion error vs. time-steps (results using Lien and Leschziner [6] and Pascau [11] formulations coincide).

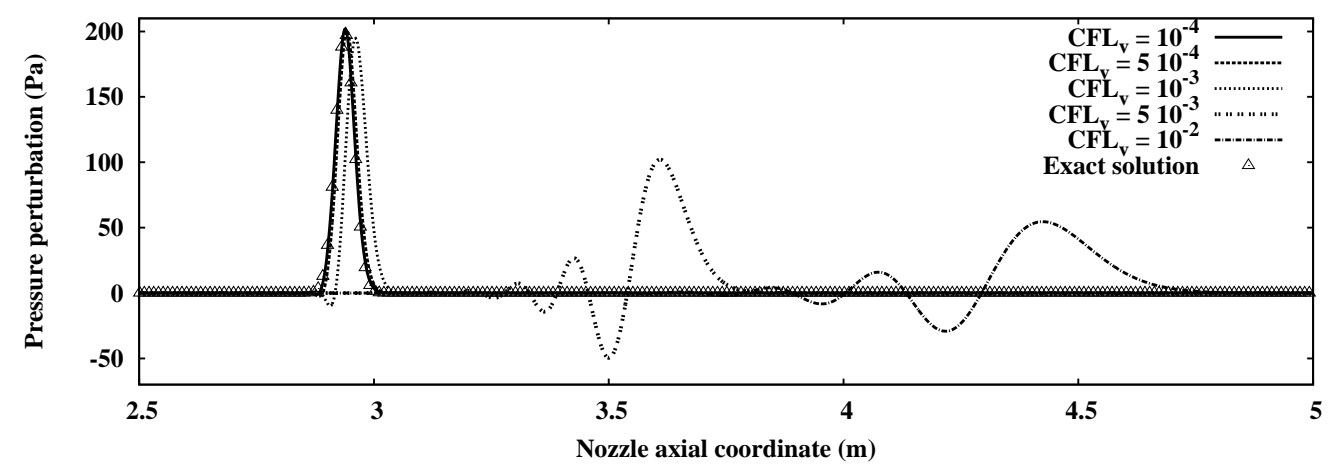

Fig. 8. Acoustic pulse propagation - Downstream propagation. Transporting velocity given in Eq. (24) (Pascau, 2011). Pressure distribution at $t=8 \mathrm{~ms}$.

made, as shown in Fig. 8. With a convective CFL less than $10^{-3}$, which corresponds in this test case to an acoustic CFL less than unity, the advection of the pulse is correctly simulated. With higher values, the simulation is not satisfying. In particular, the advection velocity is overestimated.

\subsubsection{Interference of two acoustic pulses}

A one-meter long pipe divided into $N=500$ cells is considered. The initial conditions are obtained through the method used for the acoustic pulse propagation in Sec. 4.3.2, but now, two pulses are generated in the same mean flow: one propagating to the right (downstream) and one to the left (upstream). Their initial positions 
are given by $m_{1}=0.2 \mathrm{~m}$ and $m_{2}=0.8 \mathrm{~m}$. For both pulses, $A=200 \mathrm{~Pa}$ and $s=2 \mathrm{~m}$. The mean flow is given by the values:

$$
\varrho_{0}=1.2046 \mathrm{~kg} \mathrm{~m}^{-3} \quad, \quad v_{0}=3.088610^{-3} \mathrm{~m} \mathrm{~s}^{-1} \quad, \quad p_{0}=101300 \mathrm{~Pa} .
$$

The Mach number of the mean flow is $910^{-6}$. The convective CFL number is $10^{-5}$ and the transporting velocity $v^{\mathrm{T}}$ is the one given in Eq. (24), following Pascau [11].

In Fig. 9, left, the two pulses, denoted by A and B, move towards each other. Their meeting is shown in Fig. 9, right. The interference is constructive for the density and the pressure, and destructive for the velocity. In Fig. 10, the pulses are completely overlapping. As expected, the resulting shape of the pressure is a pulse with the amplitude of $400 \mathrm{~Pa}$ (twice the amplitude of the individual pulses). In Fig. 11, the pulses are not altered by the interference. Thus, the principle of superposition of the pulses is correctly simulated. The results obtained with Eq. (23), following Lien and Leschziner [6], are quasi identical to those obtained with Eq. (24) (not shown here).
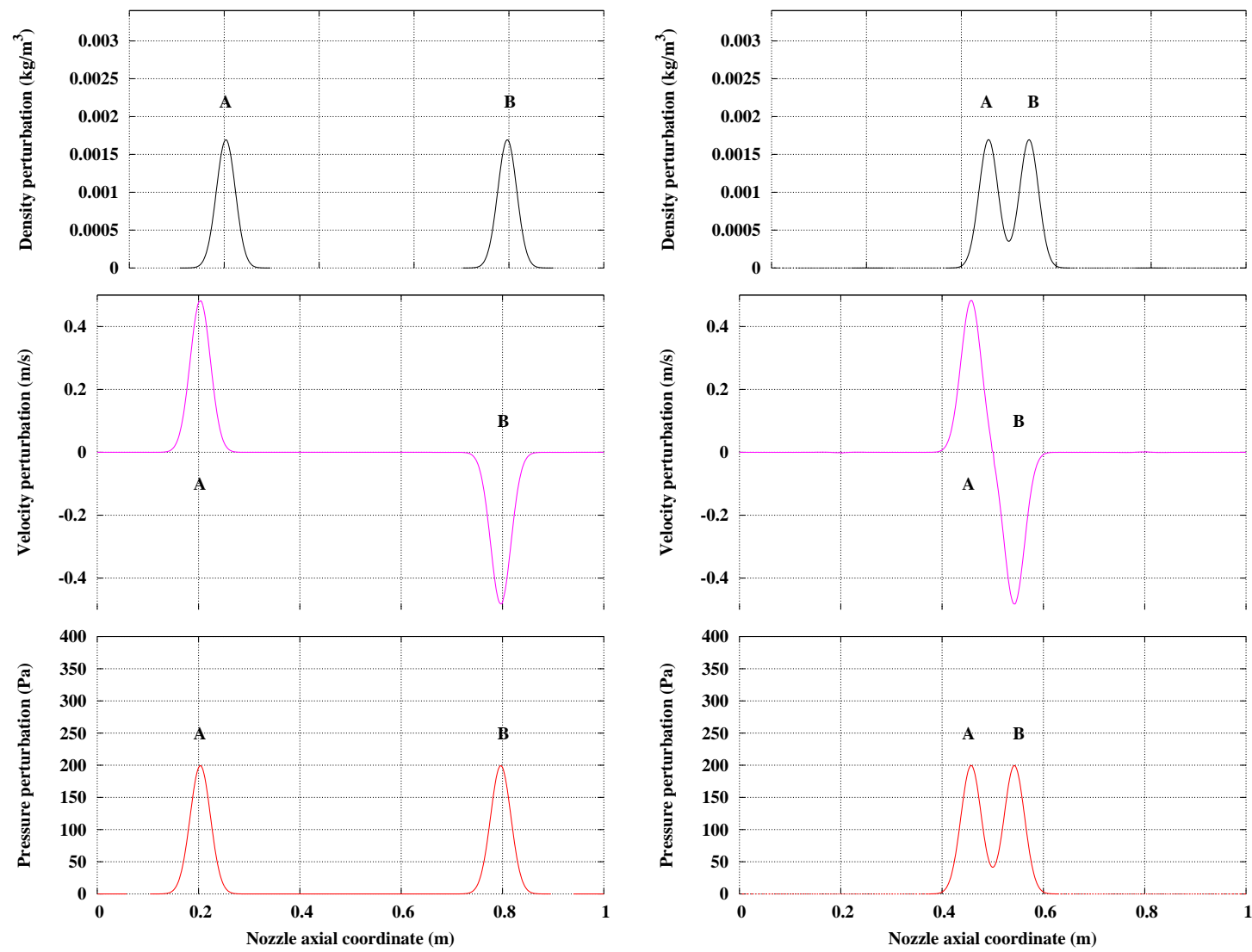

(a) $t=10^{-5} \mathrm{~s}$.

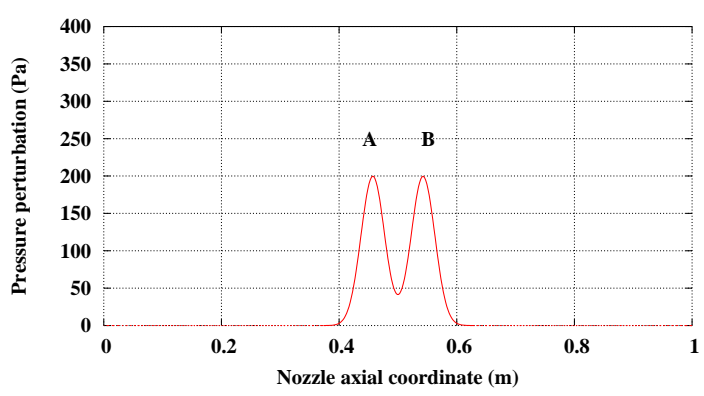

(b) $t=7.510^{-4} \mathrm{~s}$.

Fig. 9. Interference of two acoustic pulses. $\mathrm{CFL}_{v}=10^{-5}$. 

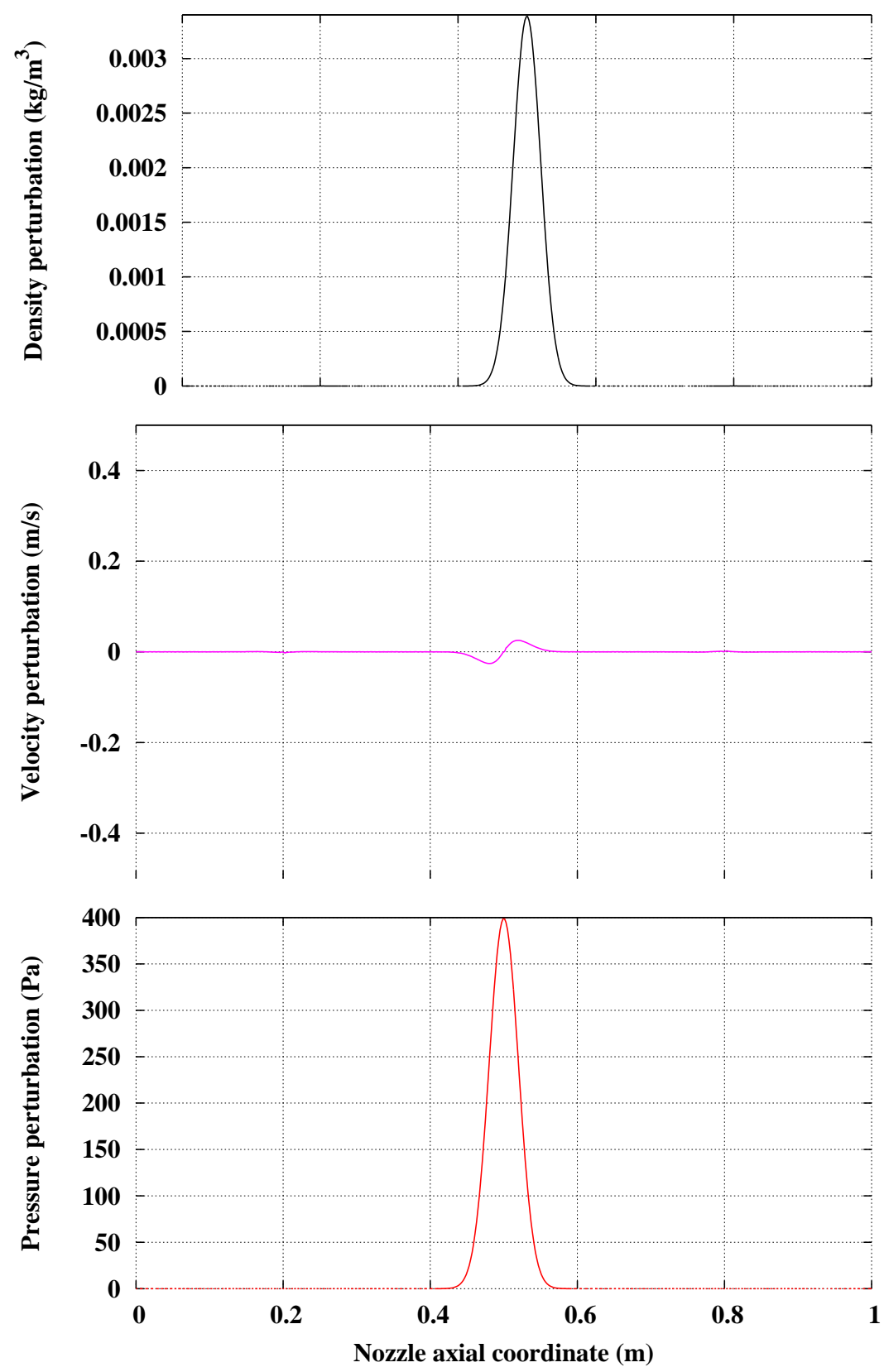

Fig. 10. Interference of two acoustic pulses at $t=8.510^{-4}$ s. $\mathrm{CFL}_{v}=10^{-5}$.

\subsubsection{Two-dimensional acoustic pulse propagation}

Up to this point, only one-dimensional test cases were considered. In order to convince the reader about its simplicity and effectiveness, the suggested approach is considered in this section for a two-dimensional test case, namely a two-dimensional acoustic pulse propagating in a low Mach number uniform flow. In cartesian coordinates, the construction of the transporting velocity from the momentum equation can be obtained dimension by dimension. For example, the first component of the transporting velocity can be constructed as follows. The first component of the mo- 

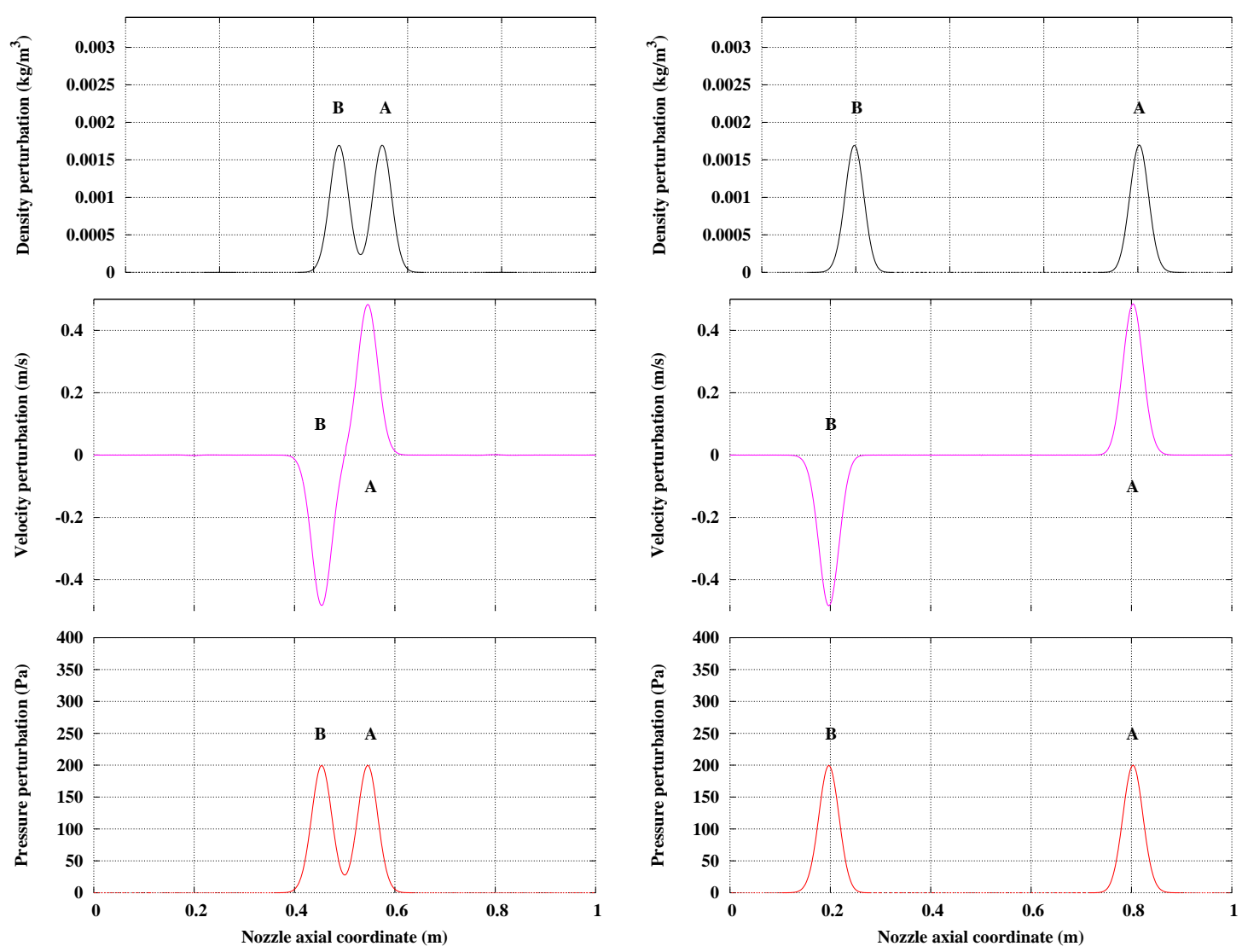

(a) $t=9.510^{-4} \mathrm{~s}$

(b) $t=1710^{-4} \mathrm{~s}$

Fig. 11. Interference of two acoustic pulses. $\mathrm{CFL}_{v}=10^{-5}$.

mentum equation reads:

$$
\partial_{t}(\varrho u)+\partial_{x}\left(\varrho u^{2}+p\right)+\partial_{y}(\varrho u v)=0
$$

where $u$ and $v$ are the velocity components in $x$ and $y$ directions, respectively. Following the discretization procedure explained in Sec. 3.2.2, we obtain, with obvious notations:

$$
B_{i j}^{u}=u_{i j}^{\star \star} A_{i j}^{u}+p_{i+1 / 2, j}^{k}-p_{i-1 / 2, j}^{k}-\frac{2 \Delta x}{\Delta t}(\varrho u)_{i j}^{n}+\frac{\Delta x}{2 \Delta t}(\varrho u)_{i j}^{n-1}+\frac{3 \Delta x}{2 \Delta t} \varrho_{i j}^{\star \star} u_{i j}^{\star \star}
$$

where

$$
A_{i j}^{u}=\varrho_{i j}^{\star \star}\left(u_{i+1 / 2, j}^{k}+\frac{\Delta x}{\Delta y} v_{i, j+1 / 2}^{k}\right)
$$


and

$$
\begin{aligned}
& B_{i j}^{u}=-\left[\frac{1}{2} \psi_{i j}\left((\varrho u)^{k}\right)\left[(\varrho u)_{i j}^{k}-(\varrho u)_{i-1, j}^{k}\right] u_{i+1 / 2, j}^{k}\right. \\
& \left.-\left\{\varrho_{i-1, j}^{\star \star} u_{i-1, j}^{\star \star}+\frac{1}{2} \psi_{i-1, j}\left((\varrho u)^{k}\right)\left[(\varrho u)_{i-1, j}^{k}-(\varrho u)_{i-2, j}^{k}\right]\right\} u_{i-1 / 2, j}^{k}\right] \\
& -\frac{\Delta x}{\Delta y}\left[\frac{1}{2} \psi_{i j}\left((\varrho u)^{k}\right)\left[(\varrho u)_{i j}^{k}-(\varrho u)_{i, j-1}^{k}\right] v_{i, j+1 / 2}^{k}\right. \\
& \left.\quad-\left\{\varrho_{i, j-1}^{\star \star} u_{i, j-1}^{\star \star}+\frac{1}{2} \psi_{i, j-1}\left((\varrho u)^{k}\right)\left[(\varrho u)_{i, j-1}^{k}-(\varrho u)_{i, j-2}^{k}\right] v_{i, j-1 / 2}^{k}\right\}\right] .
\end{aligned}
$$

The linear interpolation (see Eq. (19)),

$$
\left(\frac{B}{A}\right)_{i+1 / 2, j}^{u}=\frac{1}{2}\left(\frac{B_{i j}^{u}}{A_{i j}^{u}}+\frac{B_{i+1, j}^{u}}{A_{i+1, j}^{u}}\right)
$$

leads to the $x$-component of the interface velocity,

$$
\begin{aligned}
u_{i+1 / 2, j}^{\mathrm{T}}=\frac{1}{2 a_{i+1 / 2, j}^{u}}\left(\frac{B_{i j}^{u}}{A_{i j}^{u}}+\frac{B_{i+1, j}^{u}}{A_{i+1, j}^{u}}\right) & \\
-\frac{1}{a_{i+1 / 2, j}^{u} A_{i+1 / 2, j}^{u}}\left(p_{i+1, j}^{k}-p_{i j}^{k}\right) & +\frac{1}{a_{i+1 / 2, j}^{u} A_{i+1 / 2, j}^{u}} \frac{2 \Delta x}{\Delta t} \varrho_{i+1 / 2, j}^{n}\left(u_{i+1 / 2, j}^{\mathrm{T}}\right)^{n} \\
& -\frac{1}{a_{i+1 / 2, j}^{u} A_{i+1 / 2, j}^{u}} \frac{\Delta x}{2 \Delta t} \varrho_{i+1 / 2, j}^{n-1}\left(u_{i+1 / 2, j}^{\mathrm{T}}\right)^{n-1}
\end{aligned}
$$

where

and

$$
\frac{1}{A_{i+1 / 2, j}^{u}}=\frac{1}{2}\left(\frac{1}{A_{i j}^{u}}+\frac{1}{A_{i+1, j}^{u}}\right)
$$

$$
a_{i+1 / 2, j}^{u}=1+\frac{1}{A_{i+1 / 2, j}^{u}} \frac{3}{2 \tau} \frac{1}{\varrho_{i+1 / 2, j}^{\star \star}} .
$$

The correction step of the suggested algorithm consists in solving the following pentadiagonal system:

$$
C_{i-1, j} p_{i-1, j}^{\prime}+C_{i, j-1} p_{i, j-1}^{\prime}+C_{i j} p_{i j}^{\prime}+C_{i+1, j} p_{i+1, j}^{\prime}+C_{i, j+1} p_{i, j+1}^{\prime}=\Sigma_{i j}
$$

where

$$
\begin{aligned}
C_{i-1, j} & =-\frac{\Delta t}{\Delta x}\left[\frac{2}{3} H_{i-1 / 2, j}^{\star} \frac{\Delta t}{\Delta x}+\frac{\gamma}{\gamma-1} \mathcal{P}^{+}\left(M_{i-1, j}^{u, \star}\right) u_{i-1 / 2, j}^{\mathrm{T}}\right] \\
C_{i, j-1} & =-\frac{\Delta t}{\Delta y}\left[\frac{2}{3} H_{i, j-1 / 2}^{\star} \frac{\Delta t}{\Delta y}+\frac{\gamma}{\gamma-1} \mathcal{P}^{+}\left(M_{i, j-1}^{v, \star}\right) v_{i, j-1 / 2}^{\mathrm{T}}\right] \\
C_{i+1, j} & =-\frac{\Delta t}{\Delta x}\left[\frac{2}{3} H_{i+1 / 2, j}^{\star} \frac{\Delta t}{\Delta x}-\frac{\gamma}{\gamma-1} \mathcal{P}^{-}\left(M_{i+1, j}^{u, \star}\right) u_{i+1 / 2, j}^{\mathrm{T}}\right] \\
C_{i, j+1} & =-\frac{\Delta t}{\Delta y}\left[\frac{2}{3} H_{i, j+1 / 2}^{\star} \frac{\Delta t}{\Delta y}-\frac{\gamma}{\gamma-1} \mathcal{P}^{-}\left(M_{i, j+1}^{v, \star}\right) v_{i, j+1 / 2}^{\mathrm{T}}\right]
\end{aligned}
$$




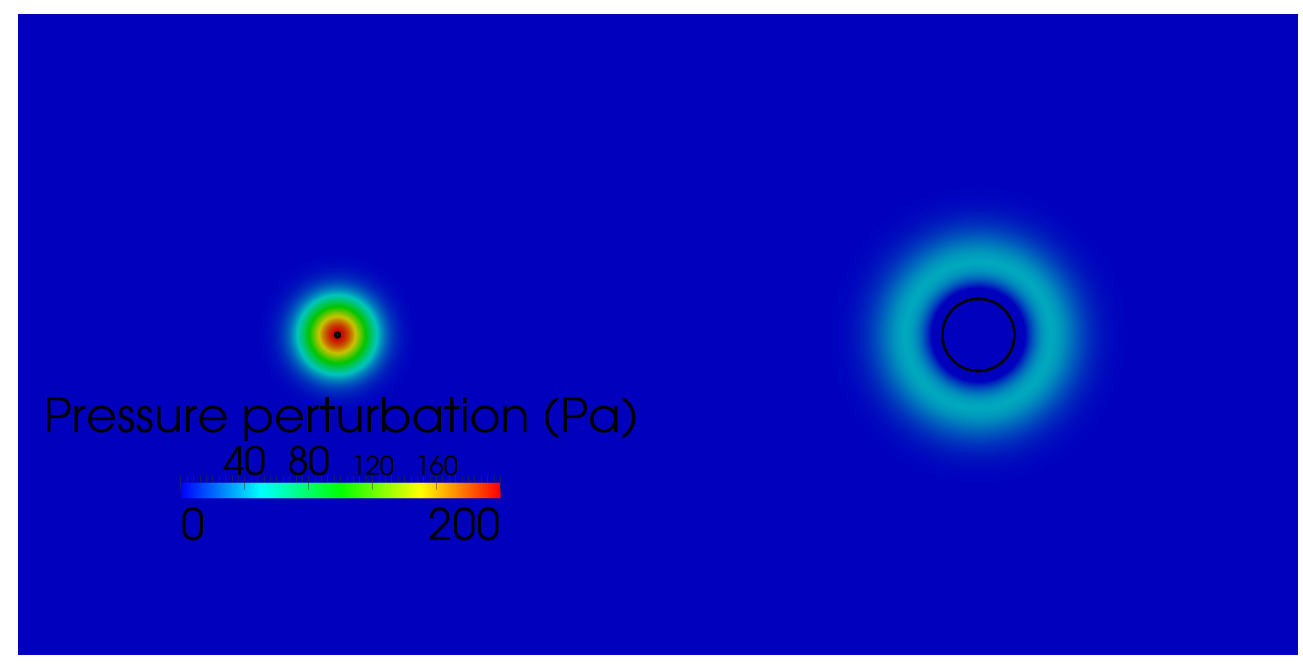

(a) $t=0$.

(b) $t=0.15 \mathrm{~ms}$.

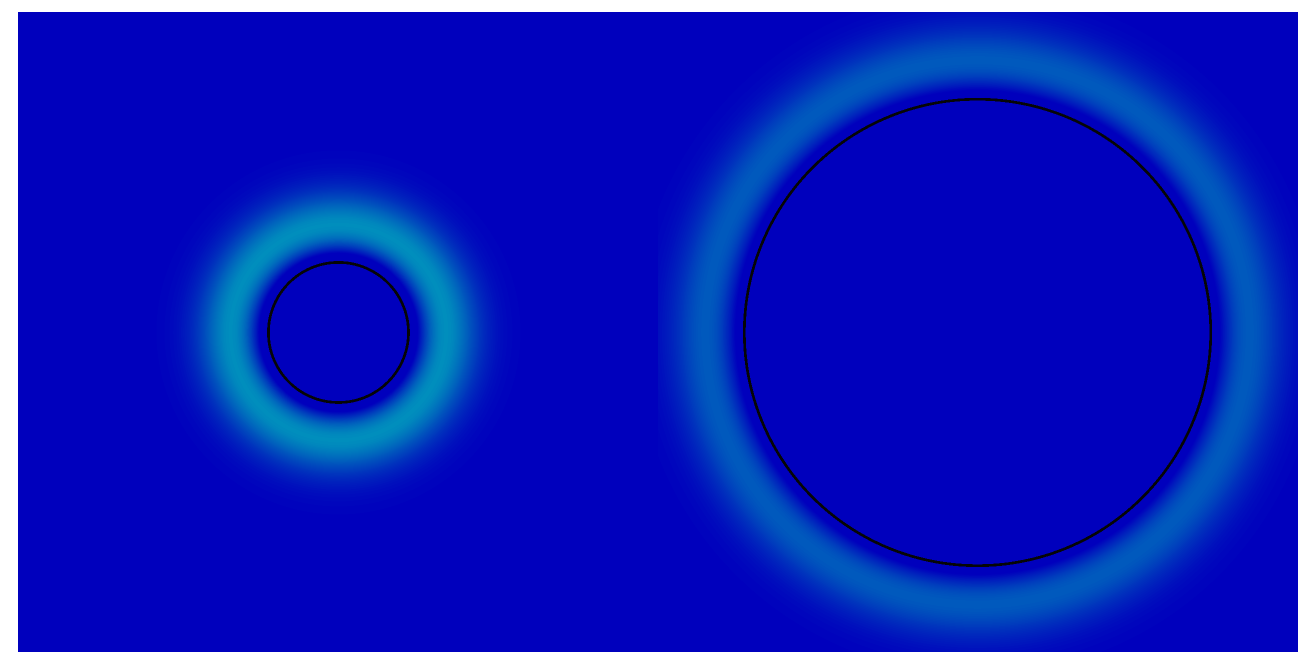

(c) $t=0.30 \mathrm{~ms}$.

(d) $t=1.00 \mathrm{~ms}$.

Fig. 12. 2-D acoustic pulse propagating in a uniform flow. Time evolution of the pressure perturbation field $(\mathrm{Pa})$ in a square of one-meter long side. Inlet: left and bottom sides of the square; outlet: right and top sides of the square. Value of the mean flow Mach number (equal in both horizontal and vertical directions): $910^{-6}$. Regular cartesian mesh, $500 \times 500$ cells. Value of the acoustic CFL number: 20. The black circle indicates the radial propagation of the black disc located in the center of the pulse at $t=0$, with the radial velocity $c_{0}=\sqrt{\gamma p_{0} / \varrho_{0}}=343.121 \mathrm{~m} / \mathrm{s}$.

$$
\begin{aligned}
& C_{i j}=\frac{3}{2(\gamma-1)}+\frac{\Delta t}{\Delta x}\left[\frac{2}{3} H_{i+1 / 2, j}^{\star} \frac{\Delta t}{\Delta x}+\frac{\gamma}{\gamma-1} \mathcal{P}^{+}\left(M_{i, j}^{\star}\right) u_{i+1 / 2, j}^{\mathrm{T}}\right. \\
& \left.+\frac{2}{3} H_{i-1 / 2, j}^{\star} \frac{\Delta t}{\Delta x}-\frac{\gamma}{\gamma-1} \mathcal{P}^{-}\left(M_{i, j}^{\star}\right) u_{i-1 / 2, j}^{\mathrm{T}}\right] \\
& +\frac{\Delta t}{\Delta y}\left[\frac{2}{3} H_{i, j+1 / 2}^{\star} \frac{\Delta t}{\Delta y}+\frac{\gamma}{\gamma-1} \mathcal{P}^{+}\left(M_{i, j}^{\star}\right) v_{i, j+1 / 2}^{\mathrm{T}}\right. \\
& \left.+\frac{2}{3} H_{i, j-1 / 2}^{\star} \frac{\Delta t}{\Delta y}-\frac{\gamma}{\gamma-1} \mathcal{P}^{-}\left(M_{i, j}^{\star}\right) v_{i, j-1 / 2}^{\mathrm{T}}\right]
\end{aligned}
$$


and

$$
\begin{aligned}
& \Sigma_{i j}=-\left\{\frac{3}{2}(\varrho E)_{i j}^{\star}-2(\varrho E)_{i j}^{n}+\frac{1}{2}(\varrho E)_{i j}^{n-1}\right. \\
& +\frac{\Delta t}{\Delta x}\left[(\varrho H)_{i+1 / 2, j}^{\star} u_{i+1 / 2, j}^{\mathrm{T}}-(\varrho H)_{i-1 / 2, j}^{\star} u_{i-1 / 2, j}^{\mathrm{T}}\right] \\
& \left.\quad+\frac{\Delta t}{\Delta y}\left[(\varrho H)_{i, j+1 / 2}^{\star} v_{i, j+1 / 2}^{\mathrm{T}}-(\varrho H)_{i, j-1 / 2}^{\star} v_{i, j-1 / 2}^{\mathrm{T}}\right]\right\} .
\end{aligned}
$$

With the dimension by dimension decomposition of the non-diagonal coefficients of the pentadiagonal system (36), an alternate direction approach procedure to solve it is straightforward and effective. This approach was employed for the simulation presented in Fig. 12, where a radially propagating two-dimensional acoustic pulse is considered. The pulse is generated by a pressure perturbation $\delta p$ and a density pertubation $\delta \varrho$ of a uniform flow which corresponds to

$$
\varrho_{0}=1.2046 \mathrm{~kg} \mathrm{~m}^{-3}, u_{0}=v_{0}=0.3088610^{-2} \mathrm{~m} \mathrm{~s}^{-1}, p_{0}=101300 \mathrm{~Pa} .
$$

Entropy fluctuations are set to zero by imposing $\delta \varrho=\delta p / c_{0}^{2}$ where $c_{0}=\sqrt{\gamma p_{0} / \varrho_{0}}$. The initial conditions are:

$$
\left\{\begin{array}{l}
\varrho^{0}=\varrho_{0}+\delta \varrho \\
u^{0}=u_{0} \quad, \quad v^{0}=v_{0} \\
p^{0}=p_{0}+\delta p
\end{array}\right.
$$

where the initial pressure pertubation is given, in $\mathrm{Pa}$, by

$$
\delta p=200 \exp \left[-\frac{(x-0.5)^{2}+(y-0.5)^{2}}{(0.05)^{2}}\right] .
$$

With an acoustic CFL number much larger than unity, $\mathrm{CFL}_{v+c}=20$, and a Mach number of the background flow around $10^{-5}$, the suggested numerical method is able to capture the acoustic wave propagation, with the correct radial velocity (see Fig. 12).

\section{Conclusion}

A pressure correction algorithm allowing unsteady calculations, including acoustics, in low Mach number regime, has been presented. Several forms of the required pressure-velocity coupling have been compared. The examples considered suggest that the strategy succeeds only if the pressure-velocity coupling, carried out through the construction of the transporting velocity, satisfies the following properties: 
- The time-step is explicitly introduced in the pressure-velocity coupling coefficient.

- The transporting velocity is defined such that the steady state is time-step independent.

\section{References}

[1] J. D. Anderson. Modern Compressible Flow: With Historical Perspective (Third Ed.). McGraw-Hill, 2004.

[2] S. K. Choi. Note on the use of momentum interpolation method for unsteady flows. Numer. Heat Transfer A, 36:545-550, 1999.

[3] S. Dellacherie. Analysis of Godunov type schemes applied to the compressible Euler system at low Mach number. J. Comput. Phys., 229:978-1016, 2010.

[4] J. R. Edwards and M.-S. Liou. Low-Diffusion Flux-Splitting Methods for Flows at All Speeds. AIAA J., 36(9):1610-1617, 1998.

[5] X. S. Li and C.W. Gu. The momentum interpolation method based on the timemarching algorithm for All-Speed flows. J. Comput. Phys., 229:7806-7818, 2010.

[6] F. S. Lien and M. A. Leschziner. A general non-orthogonal collocated finite volume algorithm for turbulent flow at all speeds incorporating second-moment turbulencetransport closure, Part 1: Computational implementation. Comput. Methods Appl. Mech. Engrg., 114:123-148, 1994.

[7] M.-S. Liou. A Sequel to AUSM: AUSM+ ${ }^{+}$. J. Comput. Phys., 129:364-382, 1996.

[8] M.-S. Liou. A Sequel to AUSM, part II: AUSM ${ }^{+}$-up for all speeds. J. Comput. Phys., 214:137-170, 2006.

[9] S. Majumdar. Role of underrelaxation in momentum interpolation for calculation of flow with nonstaggered grids. Numer. Heat Transfer A, 13:125-132, 1988.

[10] T. F. Miller and F. W. Schmidt. Use of a pressure-weighted interpolation method for the solution of the incompressible Navier-Sokes equations on a nonstaggered grid system. Numer. Heat Transfer A, 14:213-233, 1988.

[11] A. Pascau. Cell face velocity alternatives in a structured colocated grid for the unsteady Navier-Stokes equations. Int. J. Numer. Meth. Fluids, 65:812-833, 2011.

[12] T. J. Poinsot and S. K. Lele. Boundary Conditions for Direct Simulations of Compressible Viscous Flow. J. Comput. Phys., 101:104-129, 1992.

[13] C. M. Rhie and W. L. Chow. Numerical Study of the Turbulent Flow Past an Airfoil with Trailing Edge Separation. AIAA J., 21(11):1525-1532, 1983. 
[14] L. Selle, F. Nicoud, and T. Poinsot. Actual Impedance of Nonreflecting Boundary Conditions: Implications for Computation of Resonators. AIAA J., 42(5):958-964, 2004.

[15] W. Z. Shen, J. A. Michelsen, and J. N. Sørensen. Improved Rhie-Chow interpolation for unsteady flow computations. AIAA J., 39(12):2406-2409, 2001.

[16] L. L. Takacs. A Two-Step Scheme for the Advection Equation with Minimized Dissipation and Dispersion Errors. Mon. Wea. Rev., 113:1050-1065, 1985.

[17] K. W. Thompson. Time Dependent Boundary Conditions for Hyperbolic Systems. J. Comput. Phys., 68:1-24, 1987.

[18] L. N. Trefethen. Group velocity in finite difference schemes. SIAM Rev., 24(2):113136, 1982.

[19] S. Venkateswaran and C. L. Merkle. Efficiency and Accuracy Issues in Contemporary CFD Algorithms. AIAA Paper No. 2000-2251, 2000.

[20] B. Yu, W.-Q. Tao, J.-J. Wei, Y. Kawaguchi, T. Tagawa, and H. Ozoe. Discussion on momentum interpolation method for collocated grids of incompressible flow. Numer. Heat Transfer B, 42:141-166, 2001. 